\title{
Gut Dysbiosis Clinical Indicators Associate with Body Weight Gain and Cognitive Dysfunction in High-Fat Diet-Induced Pre-obese Mice
}

\author{
Paul F. Seke Etet ${ }^{1, ~ *, ~ F a y e z ~ K . ~ A l h a r b i ~}{ }^{2}$, Sayed Y. Osman ${ }^{1,3}$, Rejo Jacob Joseph ${ }^{4}$, \\ Yahia M. Bushara ${ }^{1,5}$, Lorella Vecchio ${ }^{1}$, John C. Chijuka ${ }^{2}$, Shama I. Y. Adam ${ }^{3}$, \\ Elhassan M. A. Saeed ${ }^{6,7}$, Mohammed Farahna ${ }^{1}$ \\ ${ }^{1}$ Department of Basic Health Sciences, College of Applied Medical Sciences, Qassim University, Buraydah Saudi Arabia \\ ${ }^{2}$ Department of Optometry, College of Applied Medical Sciences, Qassim University, Buraydah, Saudi Arabia \\ ${ }^{3}$ Department of Biochemistry and Molecular Biology, Faculty of Science and Technology, University of Al-Neelain, Khartoum, Sudan \\ ${ }^{4}$ Department of Medical Laboratories, College of Applied Medical Sciences, Qassim University, Buraydah, Saudi Arabia \\ ${ }^{5}$ Department of Anatomy, Faculty of Medicine, University of Al-Neelain, Khartoum, Sudan \\ ${ }^{6}$ Department of Veterinary Medicine, College of Agriculture and Veterinary Medicine, Qassim University, Buraydah, Saudi Arabia \\ ${ }^{7}$ Department of Microbiology, Faculty of Veterinary Medicine, University of Khartoum, Khartoum, Sudan
}

Email address:

paul.seke@gmail.com (P. F. Seke. Etet)

${ }^{*}$ Corresponding author

\section{To cite this article:}

Paul F. Seke Etet, Fayez K. Alharbi, Sayed Y. Osman, Rejo Jacob Joseph, Yahia M. Bushara, Lorella Vecchio, John C. Chijuka, Shama I. Y. Adam, Elhassan M. A. Saeed, Mohammed Farahna. Gut Dysbiosis Clinical Indicators Associate with Body Weight Gain and Cognitive Dysfunction in High-Fat Diet-Induced Pre-obese Mice. Journal of Diseases and Medicinal Plants. Vol. 2, No. 3, 2016, pp. $26-42$. doi: $10.11648 /$ j.jdmp.20160203.12

Received: April 18, 2016; Accepted: May 17, 2016; Published: June 16, 2016

\begin{abstract}
Obesity associates with mood and anxiety disorders, cognitive dysfunction, and motor decline, whose major causes are puzzling, since obesogenic mechanisms are complex, including a marked intestinal dysbiosis and a sustained metabolic syndrome encompassing hyperglycemia, hyperinsulinemia, systemic inflammation and oxidative stress in both humans and animal models. We addressed whether the changes occurring in mood, cognitive and motor function in dietinduced pre-obese mice are linked to intestinal dysbiosis and body weight gain. Male and female Swiss mice were made preobese by feeding with a reinforced high-fat diet for 7 weeks, along which body weight and food intake were monitored. Then, holeboard, stress-induced hyperthermia, footprint, and vertical pole tests were performed to assess cognitive and motor functions. Animals were sacrificed and intestinal dysbiosis assessed: (i) by determining fecal $\mathrm{pH}$ and gram positive bacteria counts; (ii) by assessing bacterial infiltration to the jejunum using tissue gram stain; and (iii) by assessing jejunal tissue histopathological changes (H\&E) and the immunohistochemical expression of the sustained inflammation marker CXCL10. Average increases in body weight were observed in males (37\%) and females (49\%), as well as increases in fecal $\mathrm{pH}$, gram positive counts, bacterial infiltration and CXCL10 expression, particularly marked in females. For both sexes, HFD-fed animals with more marked changes in indicators of intestinal dysbiosis also gained weight faster and displayed more marked mood, cognitive and motor alterations. These results suggest that intestinal dysbiosis is a major driver of obesity-like cognitive and motor alterations in pre-obese mice.
\end{abstract}

Keywords: High-Fat Diet, Pre-obesity, Metabolic Syndrome, Gut Bacteria, Small Intestinal Bacterial Overgrowth, Central Nervous System Functional Alteration 


\section{Introduction}

Obesity incidence has been increasing worldwide, partly due to changes in life style favoring positive energy balance and resulting excessive body weight gain, such as unhealthy eating pattern with high energy intakes, more reduced physical activity and sedentary life, particularly [1-3]. Obesity associates with mood and anxiety disorders $[4,5]$, cognitive dysfunction and dementia [6,7], and decline in both gross and fine motor skills [8, 9], whose major causes are still puzzling, since mechanisms sustaining obesity and its complications are redundant and complex. Notably, besides a sustained metabolic syndrome encompassing hyperglycemia, hyperinsulinemia, oxidative stress and systemic inflammation $[10,11]$, such mechanisms include appetite-enhancing organ- and immune-to brain signaling [12-16] and a marked intestinal dysbiosis [17, 18].

It was recently hypothesized that obesity-related metabolic syndrome may result from host genetics, diet, and gut microbiota interactions [19], as emerging clinical and experimental evidence sustains that gut microbiota imbalance (termed as dysbiosis) can trigger and fuel obesogenesis [20, 21] and obesity resistance can be induced and metabolic syndrome mitigated by diet-induced reprogramming of microbiota $[18,19,21]$, in genetically sensitive patients and experimental models. Moreover, the omics era shed new lights on the role of gut microbiota as a major immune organ, made of trillions of commensal microorganisms, which shape host homeostasis [22] and whose changes in composition or imbalance may trigger or sustain a number of diseases associated with metabolic syndrome, notably obesity-related diseases such as diabetes mellitus, inflammatory bowel disease, and gastrointestinal tract cancers [19, 21, 23], opening a promising avenue for therapeutic intervention. In the last decade, the role of dysbiosis has been extensively documented in various obesity comorbidities, including nonalcoholic fatty liver disease [16, 24], cardiovascular diseases $[18,25]$, and gastrointestinal disorders $[17,20]$. However, despite emerging evidence suggesting that intestinal dysbiosis may affect central nervous system function and play a role in the pathogenesis of neurological disorders, partly via gut-to-brain signaling [26-29], data on intestinal dysbiosis role in obesity-related mood and anxiety disorders, cognitive dysfunction, and motor skill decline are scarce.

High-fat diet-induced-obesity in mice is a model of highfat Western-diet-induced obesity, which displays sexual dimorphism in response to chronic diet consumption comparable to humans [11,30-32], including in the central nervous system [33, 34], explaining its common use in the study of pathogenic mechanisms of obesity and associated comorbidities. We have addressed changes occurring in mood, cognitive and motor function, but also in gut microbiota, in diet-induced pre-obese mice, where metabolic syndrome is less severe than in obese animals, to assess whether nervous system functional alterations and intestinal dysbiosis occur before marked metabolic syndrome.

Highlights

- Study in high-fat diet-induced pre-obese male and female mice

- Body weight gain more marked in females compared to males

- Dysbiosis more marked in HFD-fed animals gaining weight faster

- Dysbiosis more marked in females compared to males

- Dysbiosis severity correlates with marked cognitive and motor alterations

\section{Material and Methods}

\subsection{Animals and Diet}

Thirty-two adult Swiss mice (16 males weighing $28.6 \pm 3.9 \mathrm{~g}$ and 16 females weighing $27.4 \pm 2.9 \mathrm{~g}, 6$ month old) obtained from the animal house of Qassim University College of Medicine were single housed in $30 \mathrm{~cm} \times 20 \mathrm{~cm} \times$ $25 \mathrm{~cm}$ wire mesh and plastic home cages (respectively length, width and height) and monitored in a room with controlled temperature $\left(23.4^{\circ} \mathrm{C}\right)$ and $12 \mathrm{~h} / 12 \mathrm{~h}$ light-dark cycle. Experiments were started and performed in the same room after one week of acclimation. During the acclimation period animals had ad libitum access to tap water and to a low fat control diet comparable to standard mouse chow (3\% fat, $7 \%$ simple sugars, $50 \%$ polysaccharide, $15 \%$ protein [w/w], $3.5 \mathrm{kcal} / \mathrm{g}$ ), termed as "normal chow" in the present work. At the beginning of the experiment body weight was determined and two balanced groups per sex were devised: (i) a control group fed normal mouse chow $(\mathrm{n}=6)$; and $(\mathrm{ii})$ an experimental group $(\mathrm{n}=10)$ fed a high-fat diet (HFD) $(4 \%$ corn oil, $18 \%$ animal lard, $10 \%$ simple sugars, $28 \%$ polysaccharide, $23 \%$ protein $[\mathrm{w} / \mathrm{w}], \quad 4.5 \quad \mathrm{kcal} / \mathrm{g}$ ) supplemented with sweetened condensed milk (approximately $8 \%$ fat, $55 \%$ simple sugar, and $8 \%$ protein [w/w], Nestle Middle East) in an additional drinking bottle, to reach a milk and pellet daily intake of about $16 \%$ fat, $33 \%$ simple sugars and $15 \%$ protein $(\sim 4 \mathrm{kcal} / \mathrm{g})$ as reported in previous studies $[35,36]$. Such solid-liquid high-fat diet and comparable ones are established HFD known to induce obesity after 8-12 weeks [37-40].

All procedures received approval from the institutional ethical committee, and were performed in accordance with directive 2010/63/EU of the European Parliament and of the Council of 22 September 2010 on the protection of animals used for scientific purposes [41].

\subsection{Experimental Procedures}

To induce pre-obesity, mice were exposed for 7 weeks to HFD in this study. Body weight was measured twice a week and food intake every 3 days. The following behavioral tests were performed to assess changes in animals' mood, cognitive and motor functions (between 9:00 am and 12:00 pm): footprint test (exposure day 22 and ED47), holeboard 
test (ED 47), vertical pole test (ED 48), and stress-induced hyperthermia (SIH) test (ED 48). On ED45, mice were fasted for 12-h from lights on (7:00am), and fasting glycemia was determined.

All animals were sacrificed on ED 49. Visceral organs were dissected out and weighed. The gastrointestinal tract was fixed and the jejunum processed for histopathological studies (H\&E staining) and for immunohistochemical assessment of the expression of the chemokine CXCL10 (long-term inflammation marker). Bacterial infiltration in jejunal tissue was assessed using Brown and Brenn tissue gram staining, and jejunal swabs were cultured in both MacConkey and nutrient agar. Fecal $\mathrm{pH}$ and gram positive/negative counts were also determined.

\subsection{Behavioral Tests}

\subsubsection{Holeboard Test}

The holeboard test is commonly used to assess changes in emotional state in rodents as revealed by changes in exploratory activity in the holeboard arena. This ethological test presents the advantage to decrease confusion between arena exploration and general locomotor activity observed in various unconditioned tests [42], providing a more accurate evaluation of exploratory activity. The holeboard apparatus was a transparent Plexiglas box $(40 \mathrm{~cm} \times 40 \mathrm{~cm} \times 30 \mathrm{~cm})$ with 16 holes $(2.5 \mathrm{~cm}$ in diameter) cut into the floor, equidistant from the wall and from one another $(6 \mathrm{~cm}$ interval). The center of the apparatus was delimited by the 4 central holes, all located $20.5 \mathrm{~cm}$ from a corner of the box along the corner-center diagonal. A computerized digital camera was placed above the arena with a 45 degree angle to record both horizontal and vertical activities.

Testing was performed in the same room where animals were housed, in a corner with dimmed white lighting produced by fluorescent lamps ( 200 lux). Each animal was introduced, facing the wall, in a corner of the holeboard arena (always the corner closer and located in the left side of the operator). The activity in the arena was video recorded for 10 min, then animals were returned to home cages. The floor and walls of the arena were cleaned with $70 \%$ alcohol solution after each trial. Video recordings were analyzed offline using a semi-automated analysis system based on MATLAB Software Computer Vision System Toolbox (MathWorks, Natick, MA, USA). The following parameters were determined: $(i)$ the distance covered in the arena (total locomotor activity) in the first and last minutes of the trial; (ii) the latency to first head-dipping and number and duration of head dipping episodes; (iii) rearing against wall episode number (thigmotaxis [43]); (iv) the time spent in the center and corners of the arena; ( $v$ ) freezing latency and time; and (vi) grooming latency and episode number.

\subsubsection{Stress-Induced Hyperthermia Test}

Although a number of variants of SIH paradigm have been proposed based on the type of stressor [44-49], this physiological animal model of anxiety is based on the observation that mammals facing stressful events display a raise in body temperature reflecting their level of anxiety. Basically, body temperature is measured before (T1) and about $10 \mathrm{~min}$ after (T2) a mild stressor, with the difference in temperature (T2-T1) representing the $\mathrm{SIH}$ response. In the present study, animals were stressed by taking them out of their cage (after T1 measurement) and putting them on a grid positioned vertically for $10 \mathrm{~s}$. Then animals were returned to their cages where T2 was measured. Skin body temperature measured on inner ear surface (bare skin) with a handheld infrared skin thermometer (FT90, Beurer GmBH, Ulm, Germany) [50-52] was used instead of core body temperature, considering reports suggesting that (partly due to its role in thermal balancing act) skin, and particularly in the cephalic area [53], is a better indicator of behaviorallyinduced thermoregulation than core body temperature [51, 54]. SIH was expressed as the percentage of temperature before handling $(\mathrm{SIH}=(\mathrm{T} 2-\mathrm{T} 1) \times 100 / \mathrm{T} 1)$.

\subsubsection{Vertical Pole Test}

The vertical pole test is a motor task commonly used to assess changes in mouse balance, forelimb strength, as well as the ability to grasp $[55,56]$.

In this test, a hard and inflexible plastic pole (length 85 $\mathrm{cm}$, diameter $1.9 \mathrm{~cm}$ ) covered with cloth-tape and kept in vertical position by a metallic freestanding base also covered with cloth-tape, was used. Animals were placed up on the vertical pole (facing up) and video recorded until they fell or climbed off the pole. The time to first turn downward (tturn), and the total time spent on the pole (t-time), reflect of the general performance of the animals on the pole, were determined offline using the t-time score proposed by Paylor and colleagues (see table 1) [55]. The vertical pole test was performed on ED 48, just after SIH test.

Table 1. Vertical pole total time scoring system (Paylor et al., 1998).

\begin{tabular}{lll}
\hline t-time score & If the mouse: & \\
\hline 1 & Failed to grasp to the pole & \\
2 & Fell in & $0-10 \mathrm{~s}$ \\
3 & & $11-20 \mathrm{~s}$ \\
4 & & $21-30 \mathrm{~s}$ \\
5 & & $31-40 \mathrm{~s}$ \\
6 & & $41-50 \mathrm{~s}$ \\
7 & & $51-60 \mathrm{~s}$ \\
8 & Stayed on $60 \mathrm{~s}$ and climbed & halfway down \\
9 & & to the lower half \\
10 & Climbed down and off in & $51-60 \mathrm{~s}$ \\
11 & & $41-50 \mathrm{~s}$ \\
12 & & $31-40 \mathrm{~s}$ \\
13 & & $21-30 \mathrm{~s}$ \\
14 & & $11-20 \mathrm{~s}$ \\
15 & & $12-20 \mathrm{~s}$ \\
\hline
\end{tabular}

\subsubsection{Footprint Test}

Footprint test was used to assess gait disturbances in preobese mice. Footprints were obtained by allowing each mouse to walk along a runway (length $50 \mathrm{~cm}$, width $10 \mathrm{~cm}$, and $10 \mathrm{~cm}$-high Plexiglas walls, with a white paper on the floor) following feet coating with a non-toxic paint. Each animal was allowed to run once (naïve spontaneous walk), and the white paper placed on the runway floor was changed 
after each run, and the floor cleaned with $70 \%$ alcohol solution. Footprint patterns were analyzed and average stride length (distance of forward movement between strides [57]) and step length were determined (in millimeters).

\subsection{Tissue Processing, $\mathrm{H} \& \mathrm{E}$ Staining and Immunohistochemistry}

Gastrointestinal tract was dissected out, fixed in Karnovsky's fixative (5\% glutaraldehyde, $4 \%$ formaldehyde in $0.8 \mathrm{M}$ buffer) for 4 -h. Jejuna were embedded in paraffin, cut transversely (thickness $5 \mu \mathrm{m}$ ), and mounted on gelatin coated-slides. H\&E staining of transverse sections was performed following standard protocols. Stained sections were analyzed using a light microscope with a computerized video system (20× and 40× objectives), for detecting and characterizing histopathological changes.

Immunohistochemical labeling of CXC10 chemokine (also known as Interferon gamma-induced protein 10 or IP-10) was also performed using standard protocols. Briefly, transverse sections were heated for antigen retrieval, and then, deparaffinized and rehydrated (in xylene and decreasing concentration baths of ethanol). After 10 min in $\mathrm{H}_{2} \mathrm{O}_{2}(10 \%)$, sections were pre-incubated in the blocking solution (Mouse and Rabbit Specific ABC Detection Immunohistochemistry Kit, ABCAM, Cambridge, MA, USA) for $1 \mathrm{~h}$, incubated overnight in primary antibody buffer solution made of 1:200 rabbit Polyclonal IP-10 Antibody (sc-28877, Santa Cruz Biotechnology, Dallas, TX, USA) in blocking solution, and incubated for $2 \mathrm{~h}$ in secondary antibody solution (biotinylated Goat Anti-Rabbit IgG in blocking solution, ABCAM). Then, also according to kit manufacturer instructions (Mouse and Rabbit Specific ABC Detection Immunohistochemistry Kit, ABCAM), streptavidin peroxidase was applied, followed by the chromogen substrate 3,3'-diaminobenzidine hydrochloride (DAB), and hematoxylin counterstaining. Mounted sections were washed thoroughly between the steps using phosphate buffer saline $(\mathrm{pH}=7.4)$. Sections were cleared and dehydrated using graded ethanol series and xylene, and covered with a glass coverslip using DPX mounting medium (Sigma-06522, Sigma-Aldrich, St. Louis, MO, USA). CXCL10 expression was analyzed using a light microscope with a computerized video system $(20 \times$ and $40 \times$ objectives).

\subsection{Gram Staining}

\subsubsection{Fecal Gram Stain and $\mathrm{pH}$}

Fecal $\mathrm{pH}$ was determined from a solution of $200 \mathrm{mg}$ of fresh fecal material (obtained from rectal ampulla at sacrifice) in $3 \mathrm{~mL}$ of sterile distilled water. Then, smears were prepared by gentle spreading of this solution on poly-Llysine coated glass slides. After air-drying and heat fix, the bacterial smears were processed for Gram staining using a kit (Gram staining kit, CRESCENT Diagnostics, Jeddah, Saudi Arabia), following standard protocols. Gram positive and gram negative bacteria were counted systematically in 3 microscope frames $(100 \times)$ considering all bacteria in the cell and those on the top and left borders of the frame, but not lower and right borders. The average value (mean of frame values) of fecal gram positive bacteria percentage ( $\%$ of total bacteria counts that is gram positive) was determined.

\subsubsection{Brown and Brenn Tissue Gram Staining}

Brown and Brenn tissue Gram staining was performed to assess bacterial infiltration in the jejunal mucosa. Jejunal sections (5 $\mu \mathrm{m}$ thick) were deparaffinized in xylene and hydrated in decreasing alcohol concentration using standard protocols. Afterwards, sections were exposed sequentially to: (i) a $4: 1$ solution of crystal violet $(1 \%$ in distilled water, Sigma-V5265, Sigma-Aldrich, USA) and sodium bicarbonate (Hikma Pharmaceuticals, UAE) for $1 \mathrm{~min}$; (ii) gram iodine solution for $1 \mathrm{~min}$, followed by ethanol-acetone $(1: 1)$ until decolorization is complete (i.e. no more color runs off), and by basic Fuchsin solution for 1 min (gram staining kit, CRESCENT Diagnostics); (iii) solutions for differentiation reaction, i.e. brief dipping in acetone, followed by a dipping in $0.1 \%$ picric acid in acetone (until section turned yellow). After brief rinsing in acetone and acetone-xylene (1:1), sections were cleared in xylene (3 times for $5 \mathrm{~min}$ ) and mounted with DPX medium (Sigma-06522, Sigma-Aldrich). Before (iii), sections were rinsed in tap water between steps. Sections were observed on a light microscope with computerized video system $(20 \times$ and $40 \times$ objectives). CXCL10 expression was analyzed using a light microscope with a computerized video system $(20 \times$ and $40 \times$ objectives $)$. CXCL10 immunohistochemical expression was determined using densitometric analysis using MATLAB Software. The optical density of labeled sections (light adsorptance, $A_{\lambda}$ ) was calculated as follows:

$$
\mathrm{A}_{\lambda}=\left(\mathrm{I}_{0}-\mathrm{I}\right) / \mathrm{I}_{0}
$$

$\mathrm{I}_{0}$ : mean background density.

I: mean density of the labeled section

\subsection{Data Analysis}

Considering numerous reports from patient and animal model studies suggesting pivotal obesogenic roles for metabolic changes associated with (or eventually triggered by) fast body weight gain (positive energy balance) [1-3] and intestinal dysbiosis [18-21, 21], HFD animals of each sex were further divided in four groups ( $\mathrm{n}=6$ per group) to assess the impact of each of these parameters on behavioral changes indicative of cognitive and motor alterations observed in this study: two groups based on differences in body weight gain relative speed (a group made of the 6 HFD-fed animals gaining weight the slowest and conversely, a group made of the remaining 6 , i.e. animals gaining weight relatively faster), and two groups based on gram positive bacteria percentage in feces (a group made of the 6 HFD-fed animals with the lowest gram positive bacteria percentage, and conversely, a group made of the other 6 , i.e. animals with relatively high gram positive bacteria percentage). Two-tailed repeated measures ANOVA (only for comparison of ED22 and ED47 footprint test data) and two-tailed independent ANOVA (both 
followed by LSD post-hoc test) were performed to detect significant intra-parameter differences $(p<0.05)$ between all HFD-fed animals, each of the groups obtained from either body weight gain or fecal gram positive bacteria counts, and normal chow fed animals of each sex, and to assess inter-sex differences.

Data from all animals of the study were processed for multifactorial analysis, independently of experimental groups $(n=32)$ (OriginPro Software, OriginLab, Northampton, MA, USA), to assess inter-parameter interactions (as reflected by linear correlations) between body weight gain, fasting glycemia, indicators of intestinal dysbiosis (increases in fecal $\mathrm{pH}$ and fecal gram positive bacteria), and central nervous system functional changes revealed by the cognitive and motor tests performed.

\section{Results}

\subsection{General Observation and Physiological Parameters}

Major signs of central nervous system involvement were observed towards the end of the experiment, where 8 out of 10 HFD-fed males, but only 3 out of 10 females, displayed a marked increase in indicators of aggressive behavior
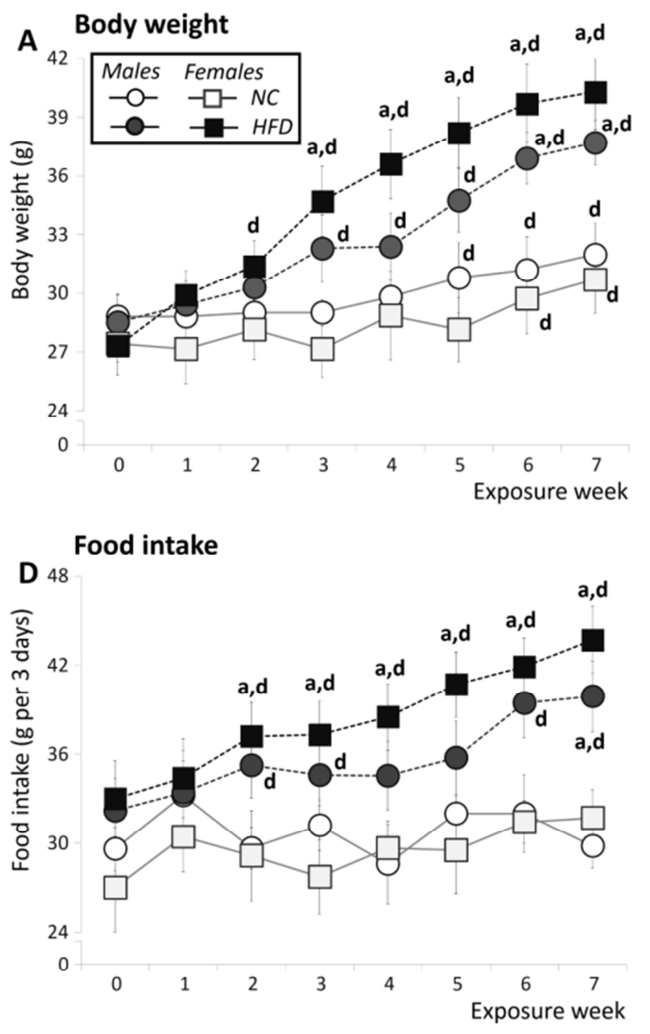

ANOVA + LSD test, p<0.05: ${ }^{a}$ vs. normal chow fed (notably: attempt to bite when handled, vocalization/squeal, jumpy). In addition, 6 out of 10 females, but no male, displayed an increase in urination with wet litter (at corners mainly) and smelly cages.

\subsubsection{Body Weight}

The progressive effects of HFD exposure on body weight are shown in figure 1A, while figure 1B presents individual total body weight gain in females and males fed either normal chow or HFD at the end of the experiment, and figure 1C shows the total body weight increase in these experimental groups. Along the 7 weeks of exposure to HFD, female mice displayed a $28.93 \%$ faster increase in body weight $\left(\mathrm{y}=1.93 \mathrm{x}+26.06, \mathrm{R}^{2}=0.97\right.$ against $\mathrm{y}=0.48 \mathrm{x}+$ $27.75, \mathrm{R}^{2}=0.89$, in females fed normal chow) than males ( $\mathrm{y}$ $=1.37 \mathrm{x}+26.60, \mathrm{R}^{2}=0.97$ against $\mathrm{y}=0.45 \mathrm{x}+26.39, \mathrm{R}^{2}=$ 0.73 , in males fed normal chow) (figure 1A). Thus, after 7 weeks of exposure, an increase in body weight higher than $30 \%$ of baseline weight was observed in $50 \%$ of male mice fed HFD, when 9 out of 10 HFD-fed females already had reached this percentage (HFD-fed females reached an increase in body weight of $30 \%$ compared to baseline weight after 4 weeks only) (figure 1B).
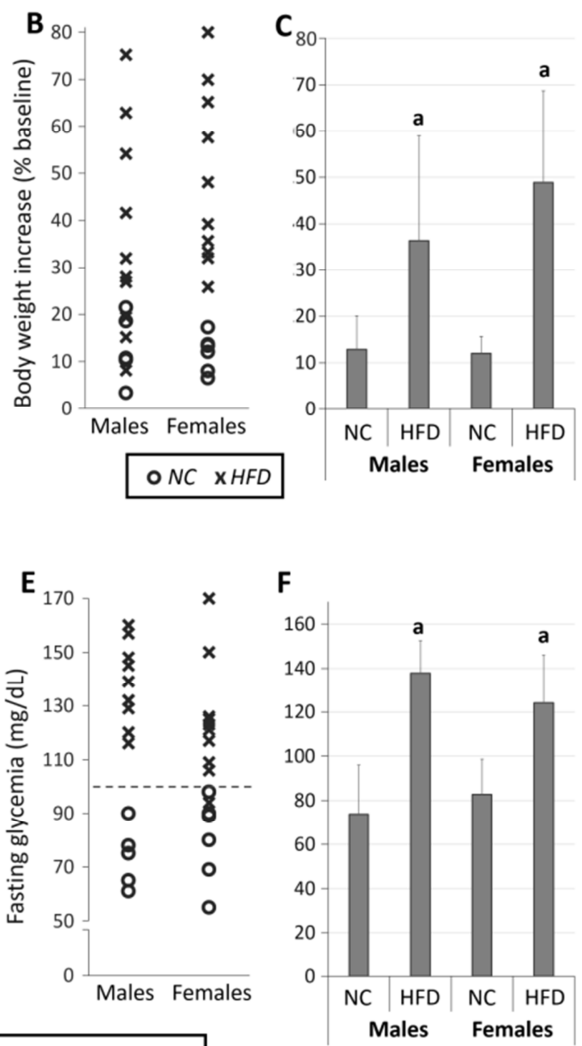

Figure 1. Physiological parameters.

A-C. Effect of high-fat diet (HFD) on body weight. A. Body weight change along the weeks of exposure. Note the significant increase from week 2 in females and week 3 in males fed HFD compared to animals fed normal chow (NC). B. Individual body weight increase. Note that the increase is faster in about $50 \%$ of animals fed HFD for both females and males. C. Total body weight increase in treatment groups. Note the significant increase in groups fed HFD. D. HFD Effects on food intake along the weeks of exposure. Note the increase in food intake with exposure. E,F. HFD effect on individual (E) and average (F) fasting glycemia. Note the increase in HFD animals and groups. Except for figures B and E, data are mean \pm standard deviation (N=10 per group). 
Body weight gain was statistically significant in HFD-fed females after 2 weeks compared to baseline values $(p=0.004)$, and after 3 weeks compared to normal chow-fed females $(p=0.002)$ (figure 1A). On the other hand, the increase in body weight was statistically significant in HFD-fed males after 3 weeks compared to baseline $(p=0.012)$, and after 6 weeks compared to normal chow-fed males $(p=0.0017)$ (figure 1A). Body weight gain was statistically significant in normal chow-fed females after 6 weeks $(p=0.0018)$ and in males after 5 weeks $(\mathrm{p}=0.034)$, compared to baseline (figure 1A). Total body weight increase was significantly higher in females $(p=0.0002)$ and males $(p=0.011)$ fed HFD compared to animals fed normal chow (figures $1 \mathrm{C}$ ).

\subsubsection{Food Intake, Fasting Glycemia and Organ Weight}

The effect of HFD on food intake along the 7 weeks of exposure is shown in figure 1D. Animals displayed an increase in food intake with HFD exposure time, with HFDfed female mice displaying a $31.1 \%$ faster increase in food intake $\left(\mathrm{y}=1.48 \mathrm{x}+31.69, \mathrm{R}^{2}=0.98\right)$ than males $(\mathrm{y}=1.02 \mathrm{x}+$ $\left.31.06, \mathrm{R}^{2}=0.84\right)$. The increase in food intake was statistically significant in HFD-fed females after 2 weeks of exposure compared to both baseline values $(\mathrm{p}=0.007)$ and to normal chow-fed females ( $p=0.021$ ) (figure 1D). Food intake increase in HFD-fed males was significant after 2 weeks of exposure compared to baseline $(\mathrm{p}=0.0005)$, and after 7 weeks compared to normal chow-fed males $(\mathrm{p}=0.014)$. No major change in food intake was observed in animals fed normal chow (figure 1D).

The effects of 7 weeks of HFD exposure on fasting glycemia are shown in figures $1 \mathrm{E}$ and $1 \mathrm{~F}$. Compared to animals of the same sex fed normal chow, fasting glycemia was significantly higher in both female $(124.1 \pm 21.9$ vs. $82.7 \pm 15.9$ in females fed normal chow, $\mathrm{p}=0.0004)$ and male $(137.8 \pm 14.7$ vs. $73.8 \pm 22.4$ in males fed normal chow, $\mathrm{p}=0.0013$ ) mice (figure 1E, F). No significant difference between females and males was observed (figure 1F), although HFD-fed males had a higher fasting glycemia (figure 1E).

The effect of HFD exposure on organ weight is shown in table 2. Gastrointestinal tract weight was increased in females (30\% increase, $\mathrm{p}=0.032)$ and males $(47.5 \%$ increase, $\mathrm{p}=0.035$ ), while liver weight was also significantly increased in females $(53.8 \%$ increase, $\mathrm{p}=0.027)$ but only slightly increased in males $(6.3 \%$ increase, $\mathrm{p}=0.797$, not significant $)$. No significant difference between genders or change in the average weight of kidneys, lungs, heart, pancreas, brain and spleen were observed in both males and females (table 2).

Table 2. Effect of high-fat diet on organ weight $(g)$.

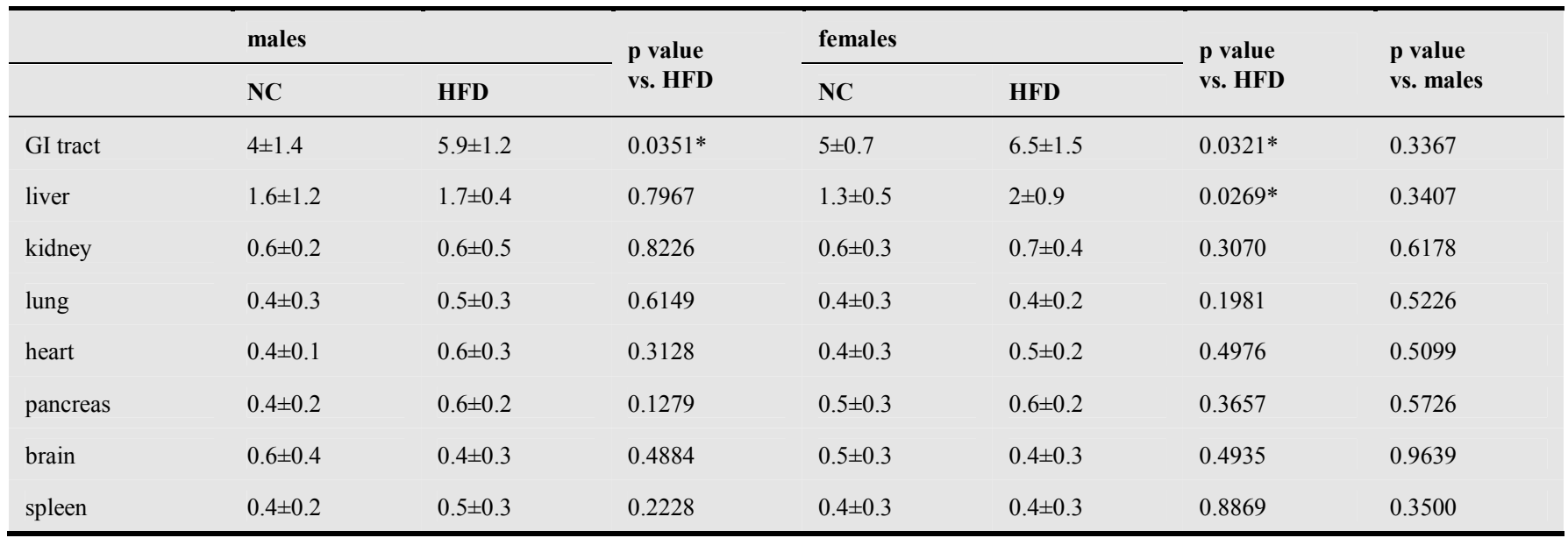

HFD: mean value for animals fed high-fat diet. $N C$ : mean value for animals fed normal chow fed. $*$ independent t test, $\mathrm{p}<0.05$. $\mathrm{N}_{\mathrm{NC}}=6$, $\mathrm{N}_{\mathrm{HFD}}=10$ males/females.

\subsection{Indicators of Intestinal Dysbiosis}

\subsubsection{Fecal Bacteria Count and pH Changes}

Figure 2 shows fecal Gram stain of representative cases of females and males fed normal chow (figures $2 \mathrm{~A}$ and $2 \mathrm{D}$ ), or HFD with either slow (figures $2 \mathrm{~B}$ and $2 \mathrm{E}$ ) or fast body weight gain (figure $2 \mathrm{C}$ and $2 \mathrm{~F}$ ) following 7 weeks of diet exposure. A decrease in gram negative bacilli was observed in animals fed HFD, concomitantly with an increase in cocci clusters and in polymorphonuclear-like leukocytes. These alterations seemed more marked in females than in males but also in HFD-fed displaying fast body weight gain than in slow weight gaining animals (figure 2).
Counts of gram positive bacteria (expressed as percentage of the sum of gram positive and gram negative bacteria counts) confirmed these observations (figure $3 \mathrm{~A}$ ), as they displayed only a slight increase in HFD-fed males with slow weight gain $(45 \%, p=0.139$, not significant) but a marked increase in females with slow weight gain $(122 \%, p=0.0035)$; and as more marked increases were observed in both fast weight gaining HFD-fed male $(97 \%, \mathrm{p}=0.0007)$ and female $(150 \%, p=0.0052)$ mice. Overall, the increase in gram positive counts was significantly higher in females compared to males $(p=0.032)$ and in fast weight gaining HFD-fed compared to slow weight gaining $(\mathrm{p}=0.028)$ (figure $3 \mathrm{~A})$. 


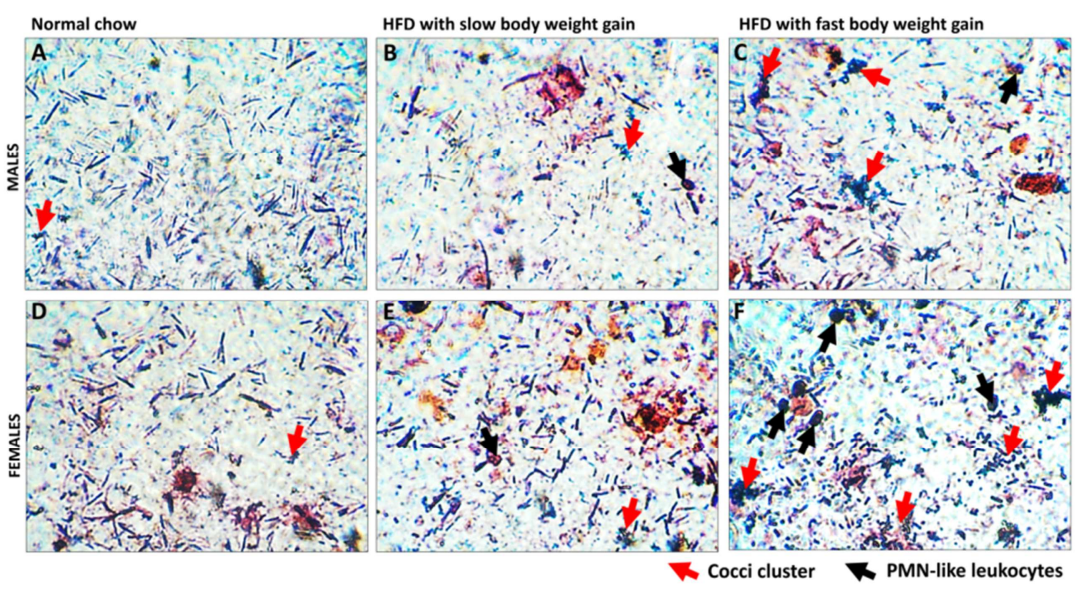

Figure 2. Fecal Gram stain micrographs.

A-C. Fecal Gram stain micrographs (100× objective) of representative cases of males fed normal chow (A), or high-fat diet (HFD) (B,C) with relatively slow (B) or fast (C) body weight gain. D-F. Fecal Gram stain micrographs of representative cases of females fed normal chow (A), or HFD (E,F) with relatively slow (E) or fast (F) body weight gain. Note the marked decrease in gram negative bacilli, and increase in cocci and in polymorphonuclear (PMN)-like leukocytes in HFD-fed females displaying fast body weight gain.

HFD exposure also associated with significant increases in fecal $\mathrm{pH}$ in females displaying either slow $(\mathrm{p}=0.017)$ or fast $(\mathrm{p}=$ 0.0056) weight gain compared to normal chow-fed animals of the same sex, but no significant change was observed in males (figure 3A). Consequently, the increase in fecal $\mathrm{pH}$ was also significantly higher in females compared to males ( $\mathrm{p}=0.032$ ).
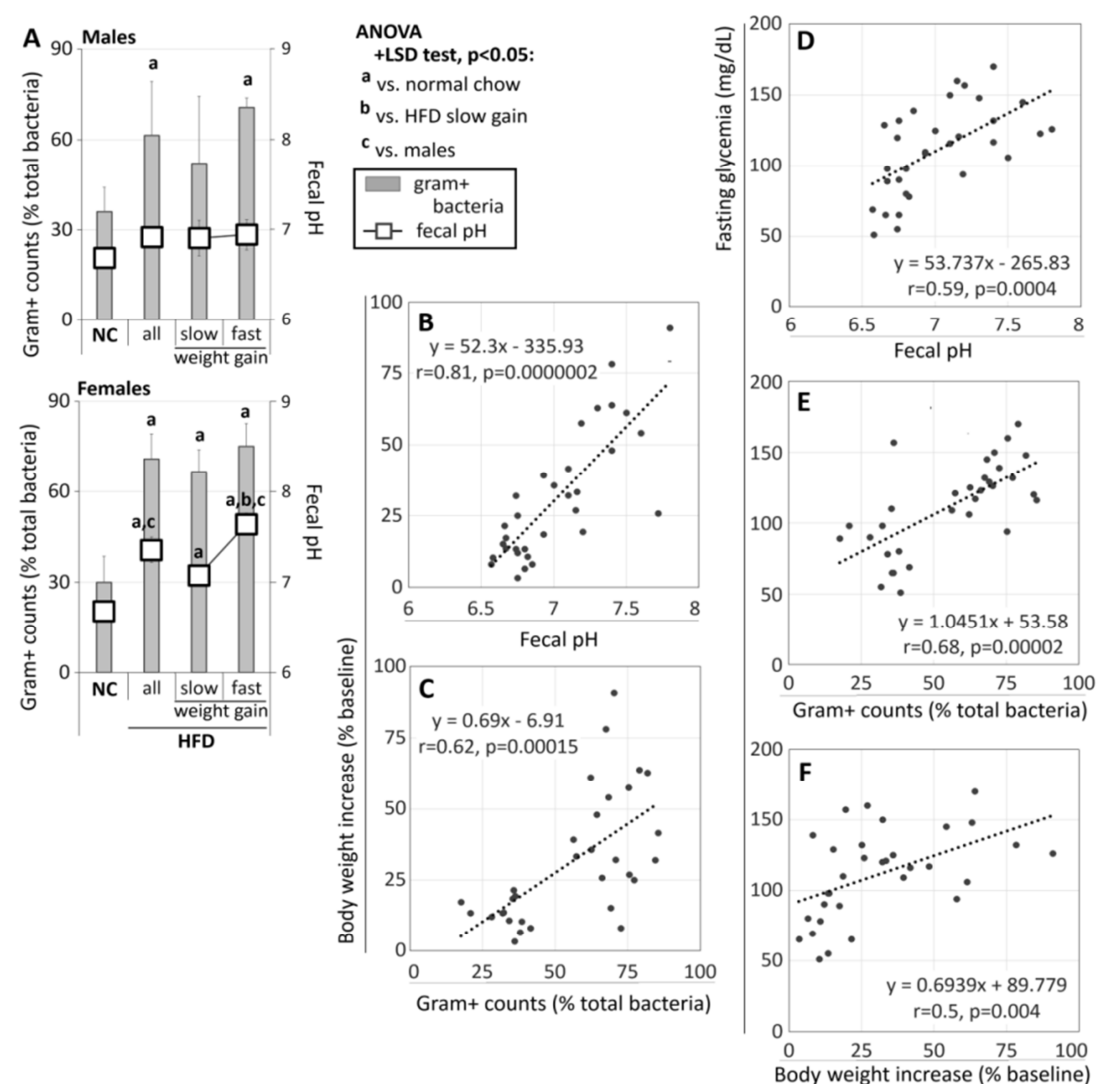

Figure 3. Fecal $\mathrm{pH}$ and bacteria counts.

A. Gram positive bacteria counts and fecal $\mathrm{pH}$ of animals either fed normal chow or fed high-fat diet (HFD). Note the increases in both parameters in HFD-fed female and male mice displaying fast weight gain. Data are mean \pm standard deviation $\left(\mathrm{N}_{\mathrm{NC}}=10, \mathrm{~N}_{\mathrm{HFDall}}=12, \mathrm{~N}_{\mathrm{HFDslow} / \text { ast }}=6\right)$. B,C. Body weight gain in function of fecal $\mathrm{pH}(\mathrm{B})$ or fecal gram positive bacteria counts (C). D-F. Fasting glycemia in function of fecal $\mathrm{pH}$ (D), fecal gram positive bacteria counts (E), and body weight gain $(\mathrm{F})$. Note the positive correlations. Points represents individual values $(\mathrm{N}=32)$. 
Interestingly, significant positive correlations were observed between total body weight increase and fecal $\mathrm{pH}$ $(\mathrm{y}=52.3 \mathrm{x}-335.93, \mathrm{r}=0.81$ ) (figure $3 \mathrm{~B})$, and between total body weight increase and fecal gram positive bacteria counts $(\mathrm{y}=0.69 \mathrm{x}-6.91, \mathrm{r}=0.62)$ (figure 3C). Although in a lesser extent, fecal $\mathrm{pH}(\mathrm{y}=53.74 \mathrm{x}-265.83, \mathrm{r}=0.59)$ (figure 3D) and fecal gram positive bacteria counts $(\mathrm{y}=1.05 \mathrm{x}+53.58, \mathrm{r}=0.68)$ (figure $3 \mathrm{E}$ ) also correlated positively with fasting glycemia, which correlated with total body weight increase as well $(y=0.69 x+89.78, r=0.62)$ (figure $3 F)$.

\subsubsection{Jejunal Swab Cultures}

Figures $4 \mathrm{~A}-\mathrm{K}$ show the result of cultures in both nutrient (figures $4 \mathrm{~A}-\mathrm{E}$ ) and MacConkey agar (figures $4 \mathrm{G}-\mathrm{K}$ ) of jejunal swabs of 3 randomly selected animals from each group of study (normal chow-fed, HFD-fed displaying slow body weight gain, HFD-fed displaying fast body weight gain). Nutrient agar cultures revealed: (i) small white bacterial colonies in all groups in males, while in females only in normal chow-fed and HFD-fed displaying slow weight gain (not in HFD-fed displaying fast weight gain); (ii) large white mucoid bacterial colonies in all HFD-fed groups but not in normal chow-fed; and (iv) fungal colonies in all animals (figures 4A-E). Gram staining of a large white mucoid bacterial colony revealed gram positive cocci in clusters (figure 4F).
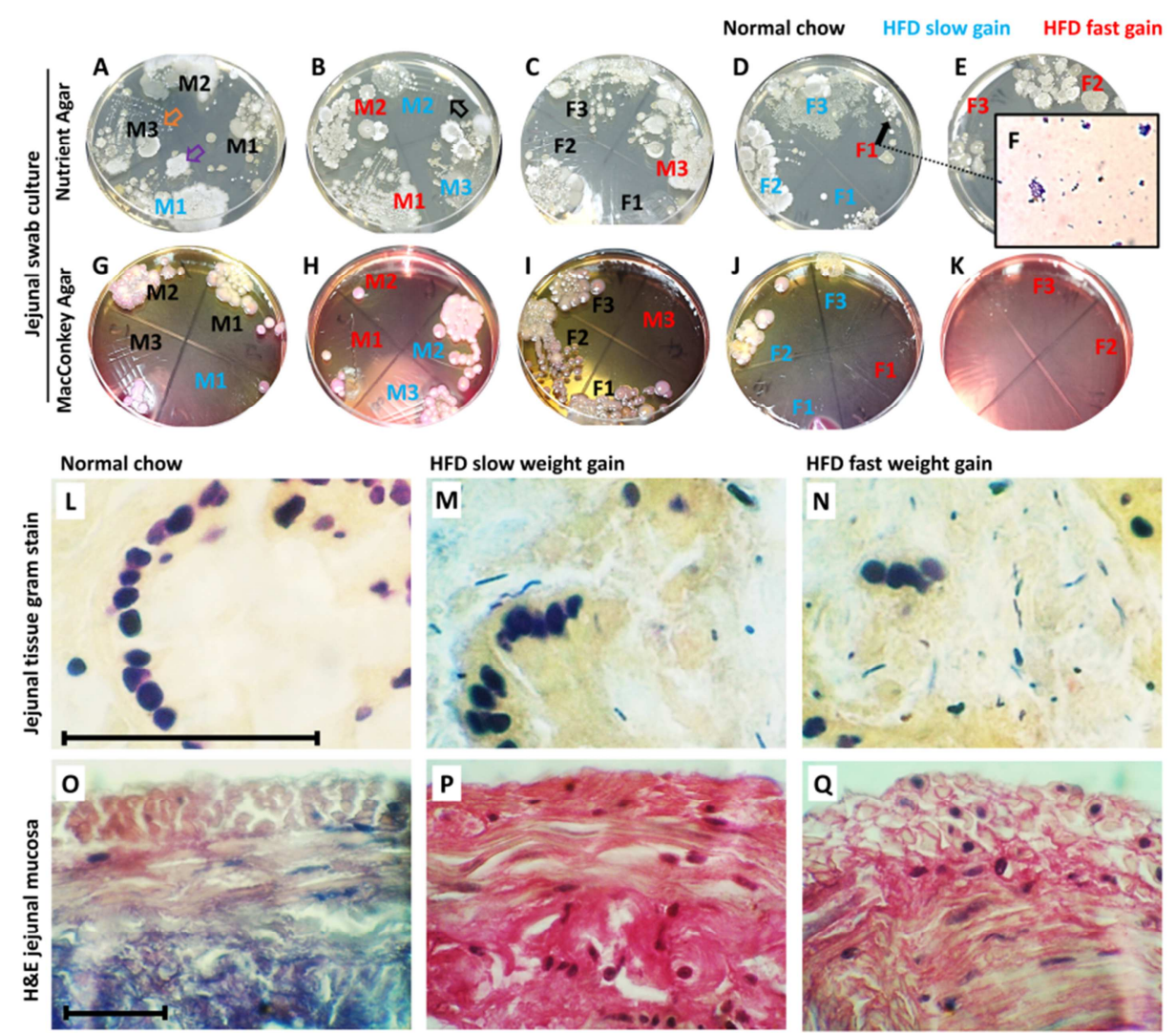

HFD slow weight gain
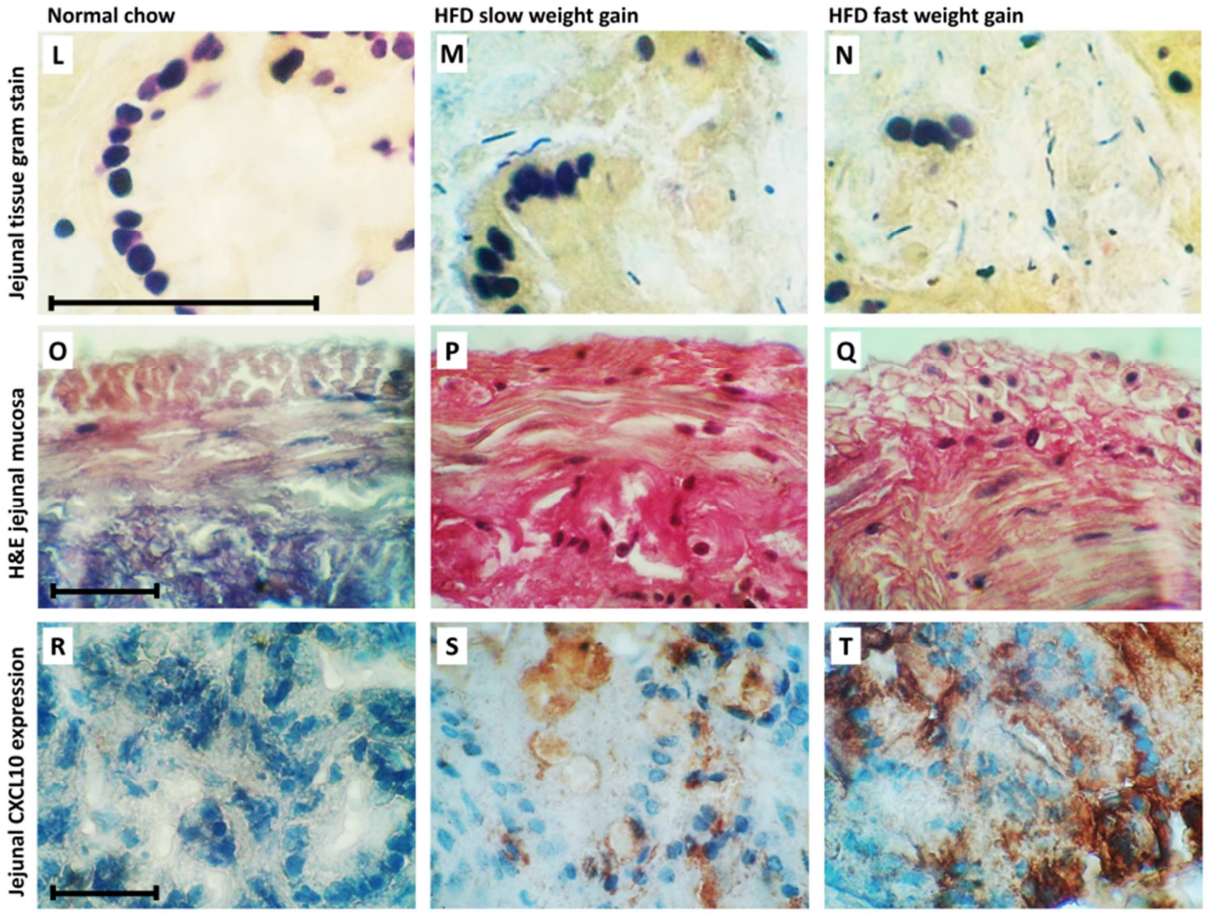

Figure 4. Jejunal changes.

A-E. Jejunal swab cultures in nutrient agar. Note the absence of small white bacterial colonies (orange empty arrow) in high-fat diet (HFD)-fed females displaying fast weight gain, the presence of large white mucoid bacterial colonies (black empty arrow) in all HFD-fed groups except in normal chow-fed, and the presence of fungal colonies in all animals (violet empty arrow). F. Gram staining micrograph of a small colony (black plain arrow head in D) collected from nutrient agar culture of a female fed HFD displaying fast weight gaining showing clusters of cocci. G-K. Jejunal swab cultures in MacConkey agar. Note the coliform (Klebsiella-like) bacteria growth (large mucoid pink colonies) in most groups, with a decrease (and then non-growth) in HFD-fed animals with increasing body weight gain. L-N. Brown and Brenn gram stain of jejuna of representative cases of HFD-fed and normal chow-fed females. Note the infiltrating bacilli in HFD-fed animals. O-Q. H\&E micrographs of the same animals. Note the degeneration of epithelial cells and tissue fluid exudate in the jejunal mucosa of HFD-fed animals. R-T. CXCL10 expression in jejuna of the same animals (immunohistochemistry +hematoxylin stain). Note the increase in CXCL10 expression in animals fed HFD. Scale bars: $100 \mu \mathrm{m}$. 
On the other hand, large mucoid lactose fermenting coliform (Klebsiella-like) colonies were observed in MacConkey agar cultures of swabs from all groups (figures $4 \mathrm{G}-\mathrm{J}$ ), except those from HFD-fed females displaying fast weight gain, where no growth was observed (figures $4 \mathrm{~J}, \mathrm{~K}$ ). Notably, a marked decrease in the number of colonies (compared to normal chow-fed) was observed in HFD-fed females displaying slow weight gain (figure $4 \mathrm{~J}$ ) as well as in HFD-fed males displaying fast weight gain (figures $4 \mathrm{H}, \mathrm{I}$ ), but not in HFD-fed males displaying slow weight gain that displayed normal number of mucoid colonies (i.e. comparable to normal chow) (figures $4 \mathrm{G}, \mathrm{H})$.

\subsubsection{Tissue Gram Stain, Histopathological Changes and CXCL10 Expression}

Figures $4 \mathrm{~L}-\mathrm{N}$ present Brown and Brenn gram stain of jejuna of representative cases of a normal chow-fed female (figure 4L) and of HFD-fed females with slow (figure 4M) or fast (figure $4 \mathrm{~N}$ ) body weight gain. Infiltrating bacilli were observed in jejuna of all HFD-fed females displaying fast weight gain and in most ( 3 out of 5) females displaying slow weight gain. Infiltrating bacilli were also present in most (3

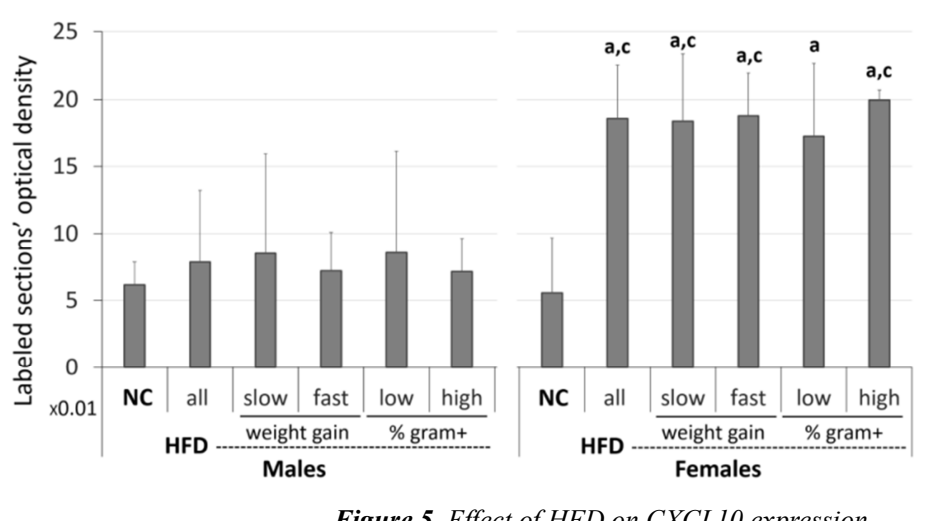

Figure 5. Effect of HFD on CXCL10 expression. of 5) HFD-fed males displaying fast weight gain, while no infiltration was observed in HFD-fed males displaying slow weight gain.

Histopathological analysis of H\&E stained jejunal sections did not revealed any major change in HFD-fed females displaying a slow weight gain (figure 4P) compared to females fed normal chow (figure 4O). However, signs of non-specific inflammation were observed in the jejunal mucosa of HFD-fed females displaying fast weight gain, notably, marked tissue fluid exudate (edema) and degeneration of epithelial cells (figure 4Q). No major change was observed in HFD-fed males.

Immunohistochemical analysis of CXCL10 expression in jejuna of the same animals revealed no expression in normal chow-fed (figure 4R), a low expression in HFD-fed females with slow weight gain (figure 4S), and a marked expression in HFD-fed females with fast weight gain (figure 4T). In males, only a slight increase in CXCL10 expression was observed in HFD-fed animals with fast weight gain. These observations were confirmed by densitometric analysis (figure 5).
Note the increase in CXCL10 expression in animals treated with high-fat diet (HFD), compared to those receiving normal chow (NC).

\subsection{Cognition, Mood and Motor Function Assessment}

\subsubsection{Holeboard Test}

The effect of 7 weeks of HFD exposure on holeboard test parameters are shown in figures 6A-G.

\section{Distance covered in the holeboard arena}

Figure 6A shows the distances covered the first and last minutes spent in the arena (expressed as the percentage of the total distance covered in the maze) as well as their ratio. The distance covered the first minute was slightly increased in HFD-fed males displaying low weight gain (10.51\% increase, not significant), but significantly decreased in those displaying fast gain $(35.4 \%$ decrease, $\mathrm{p}=0.0476$ vs. males fed normal chow; $41.78 \%$ decrease, $\mathrm{p}=0.0345$ vs. slow weight gaining HFD-fed males). No other marked change was observed in this parameter in any of the other groups.

The distance covered in the last minute was increased in females $(43.68 \%$ increase, $\mathrm{p}=0.0094)$ and males $(52.94 \%$ increase, $p=0.0049$ ) fed HFD, compared to animals fed normal chow (figure 6A). Division by weight gaining speed revealed significant increases only in slow weight-gaining HFD-fed males $(111.4 \%$ increase, $p=0.0037)$. However, divisions made based on fecal gram positive bacteria counts revealed significant increases in both males and females with high counts $(124.82 \%$ increase, $\mathrm{p}=0.0367$ and $98.8 \%$ increase, $\mathrm{p}=0.0031$, respectively), but not in groups with low counts.

The ratio of the distance covered in the last minute to the distance covered in the first minute was also increased in HFD-fed females $(41.82 \%$ increase, $\mathrm{p}=0.031)$ and males $(58.73 \%$ increase, $\mathrm{p}=0.026)$ compared to animals fed normal chow (figure 6A). Increases observed in HFD-fed animals were more marked in groups with fast weight gaining and high fecal gram positive bacteria counts. However, a statistically significant increase was observed only in females with high fecal bacteria counts $(55.97 \%$ increase, $\mathrm{p}=0.023$ vs. females fed normal chow), due to high inter-individual variability in the other groups. 


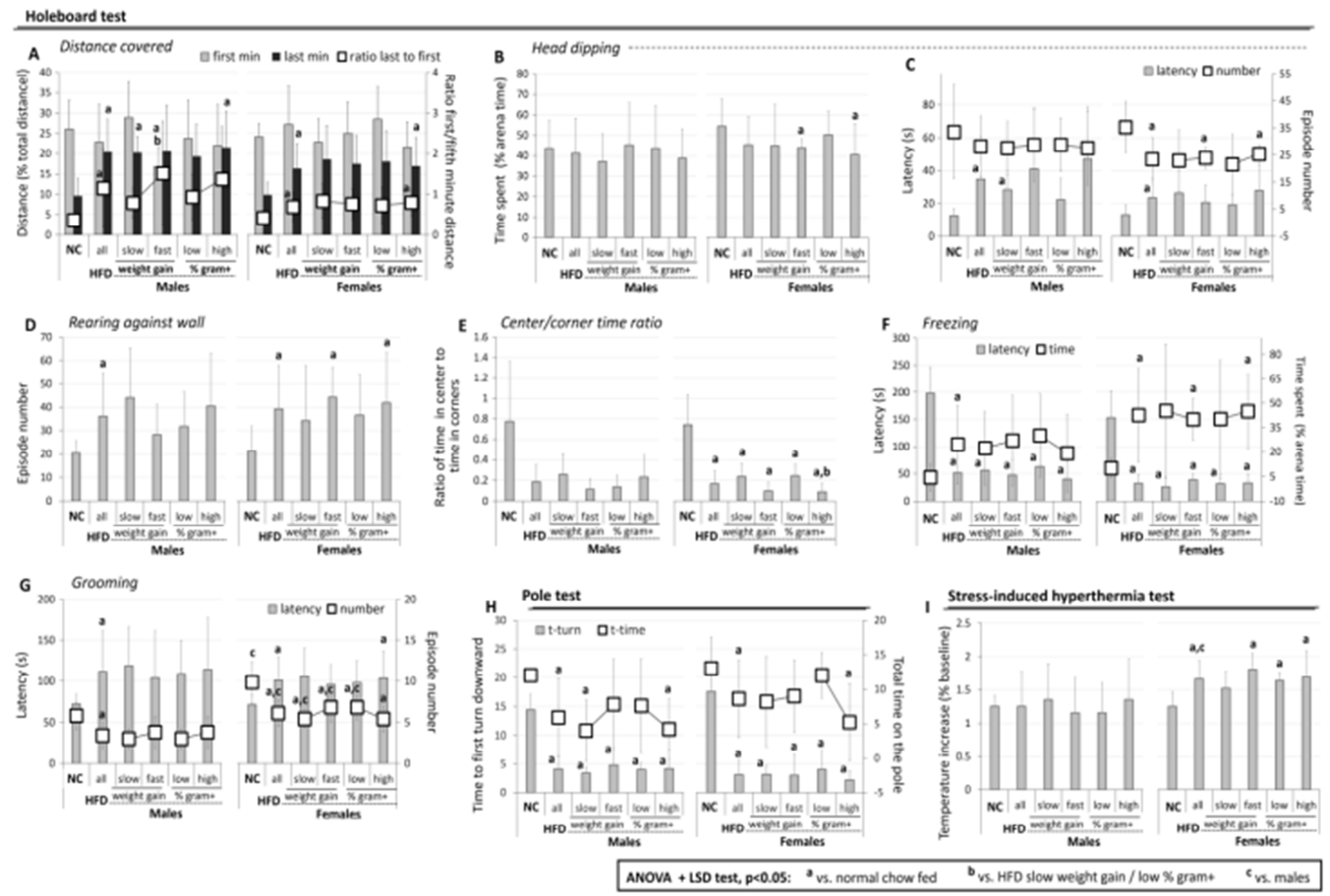

Figure 6. Cognitive and motor tests.

A-G. Holeboard test parameters. A. Distance covered in the first and last minute spent in the arena, and ratio of the distance covered in the last to the distance covered in the first minute. Note the increases in last minute distance and in ratio in females and males fed high-fat diet (HFD) compared to animals fed normal chow (NC). B,C. Head dipping time (B), latency and episode number (C). Note the decrease in episode number in animals fed HFD. D. Rearing against wall episode number. Note the marked increase in females fed HFD. E. Ratio of time spent in the center and in the corners of the holeboard arena. Note the marked decrease in females fed HFD. F. Freezing latency and time. Note the decreased latency and increased time in HFD-fed groups. G. Grooming latency and episode number. Note the higher episode number in females and the increased latency in both females and males fed HFD. H. Vertical pole total time (t-time) and time to first turn downward (t-turn). Note the decreases in both parameters in HFD-fed groups. I. Stress-induced hyperthermia (SIH) test. Note SIH increase in females fed HFD. Data are mean \pm standard deviation.

\section{Head dipping latency, episode number, and time}

HFD exposure decreased head dipping time (figure 6B), but such decrease was statically significant only in females with fast weight gain $(21.59 \%$ decrease, $\mathrm{p}=0.036$ vs. females fed normal chow) or high fecal gram positive bacteria counts ( $35.55 \%$ decrease, $\mathrm{p}=0.025$ vs. females fed normal chow).

HFD exposure increased head dipping latency in both females $(44.68 \%$ increase, $\mathrm{p}=0.027$ vs. females fed normal chow) and males $(63.69 \%$ increase, $\mathrm{p}=0.032$ vs. males fed normal chow) (figure 6C). Increases were observed in all HFD-fed groups (more marked in animals with fast weight gain or with high fecal gram positive bacteria counts), but a significant increase was observed only in males with slow weight gain $(103.08 \%$ increase, $\mathrm{p}=0.0301$ vs. males fed normal chow), as other groups displayed a high interindividual variability.

Significant decreases in head dipping episode number were observed in HFD-fed females ( $50.21 \%$ decrease, $\mathrm{p}=0.02$ vs. females fed normal chow) but not males (19.14\% decrease, $\mathrm{p}=0.551$ vs. males fed normal chow) (figure 6C). Such decreases were more marked in females with fast weight gain $(58.48 \%$ decrease, $\mathrm{p}=0.021)$ or high fecal gram positive bacteria counts $(64.55 \%$ decrease, $\mathrm{p}=0.035)$. No significant difference in head dipping latency, episode number, and time was observed between genders (figures 6B,C).

\section{Rearing against wall episode number}

HFD exposure increased the number of episodes of rearing against wall in both females $(46.17 \%$ increase, $p=0.004)$ and males ( $44.04 \%$ increase, $p=0.0287$ ), compared to animals of the same genders fed normal chow (figure 6D). Increases were more marked in animals with high fecal gram positive bacteria counts, particularly in females $(117.78 \%$ increase, $p=0.0292)$. Females, but not males, with fast weight gain also displayed an 
increase $(114.86 \%$ increase, $\mathrm{p}=0.0129)$. No significant difference was observed between genders (figure 6D).

\section{Time in arena center and corners}

HFD exposure decreased the ratio of the time spent in the center to the time spent in the corners of the holeboard arena (figure 6E). Such decrease was only statistically significant in females $(71.43 \%$ decrease, $p=0.0014$ vs. females fed normal chow), where marked decreased were observed in animals displaying fast weight gain $(88.86 \%$ decrease, $\mathrm{p}=0.0008$ vs. females fed normal chow) or high fecal gram positive bacteria counts $(88.1 \%$ decrease, $p=0.0007$ vs. females fed normal chow, and $63.99 \%$ decrease, $\mathrm{p}=0.0387$ vs. HFD-fed females with low counts). No significant difference was observed between genders (figure 6E).

\section{Freezing latency and time}

HFD exposure decreased freezing latency in both females ( $78.89 \%$ decrease, $\mathrm{p}=0.0004$ vs. females fed normal chow) and males $(73.89 \%$ decrease, $\mathrm{p}=0.0007$ vs. males fed normal chow) with no major differences between fast and slow weight-gaining HFD-fed animals, animals with low or high gram positive fecal bacteria counts, or between genders (figure 6F).

Increased total freezing time were also observed in both HFD-fed females $(76.76 \%$ increase, $p=0.0057$ vs. females fed normal chow) and males (82.93\% increase, $p=0.033$ vs. males fed normal chow) (figure $6 \mathrm{~F}$ ). However, increases with fecal gram positive bacteria counts-based divisions, but not in weight gaining speed-based divisions, were accompanied by increases in total freezing time. Nonetheless, significant increases were observed in HFD-fed females with high fecal gram positive bacteria counts $(350 \%$ increase, $\mathrm{p}=0.0254)$ and in those displaying fast weight gain $(300 \%$ increase, $\mathrm{p}=0.0042$ ) compared to females fed normal chow. No significant difference was observed between genders (figure $6 \mathrm{~F})$.

\section{Grooming latency and episode number}

HFD exposure increased grooming latency in both females ( $29.04 \%$ increase, $p=0.011$ vs. females fed normal chow) and males $(35.19 \%$ increase, $\mathrm{p}=0.039$ vs. males fed normal chow) (figure 6G). Although no significant difference based either on body weight gain speed or on fecal gram positive bacteria counts was observed in HFD-fed males, in females, grooming latency increase was more marked in animals with high fecal gram positive bacteria counts $(45.03 \%$ increase, $\mathrm{p}=0.0123$ vs. females fed normal chow). No significant difference in grooming latency was observed between genders (figure 6G).

On the other hand, HFD exposure decreased grooming episode number in both females $(62.3 \%$ decrease, $\mathrm{p}=0.0068$ vs. females fed normal chow) and males $(70.59 \%$ decrease, $\mathrm{p}=0.028$ vs. males fed normal chow) (figure 6G). No significant difference in grooming episode number was observed between fast and slow weight gaining, or low and high fecal gram positive bacteria counts, in HFD-fed males (figure 6G). However, HFD-fed females displayed significant decreases compared with normal chow fed females, more marked with fecal gram positive bacteria counts, but not weight gaining speed (figure 6G). Interestingly, females displayed significantly higher grooming episode numbers independently of the diet $(44.26 \%$ higher, $\mathrm{p}=0.0013$ for females fed HFD and $41.41 \%$ higher, $\mathrm{p}=0.0074$ for females fed normal chow), compared to males receiving the same diet (figure 6G).

\subsubsection{Vertical Pole Test}

The effect of HFD exposure on vertical pole test parameters, i.e. time to first turn downward (t-turn) and total time on the vertical pole (t-time) are shown in figure $6 \mathrm{H}$. HFD exposure induced decreases in t-turn in females ( $82.39 \%$ decrease, $p=0.0062$ vs. females fed normal chow) and males $(71.53 \%$ decrease, $\mathrm{p}=0.0002$ vs. males fed normal chow), with no difference based on body weight gain speed or fecal gram positive bacteria counts.

Decreases were also observed in t-time in HFD-fed females $(46.52 \%$ decrease, $\mathrm{p}=0.006$ vs. females fed normal chow) and males $(64.02 \%$ decrease, $\mathrm{p}=0.0001$ vs. males fed normal chow) (figure $6 \mathrm{H}$ ). Unlike divisions based on body weight gain speed, t-time decreases were more marked with fecal gram positive bacteria counts in both females $(60 \%$ decrease, $\mathrm{p}=0.016$ in HFD fed with high counts vs. females fed normal chow) and males ( $65 \%$ decrease, $p=0.035$ in HFD fed with high counts vs. males fed normal chow). No significant difference was observed between genders in t-turn and t-time (figure $6 \mathrm{H}$ ).

\subsubsection{Stress-Induced Hyperthermia Test}

The effect of HFD exposure on SIH (the body temperature increase observed following mild stress) is shown in figure 6I. SIH was more marked in females fed HFD as compared with both females fed normal chow $(29.41 \%$ increase, $\mathrm{p}=0.0041)$ and with HFD-fed males $(23.53 \%$ increase, $\mathrm{p}=0.045)$. SIH was increased with fecal gram positive bacteria counts in both HFD-fed males and females, whereas SIH was increased with body weight gain speed in HFD-fed females, but not males (figure 6I).

\subsubsection{Footprint Analysis}

Figures 7. A-C show footprints of representative cases of normal chow-fed (figure 7A), and of a HFD-fed animal at exposure day (ED) 22 (figure 7B) and ED47. The analysis of footprints revealed a tendency to stand on toes only, and shorter steps compared to normal chow-fed animals. The latter observation was confirmed step (figure 7D) and stride length (figure 7E) measurement, which confirmed significant reductions in both females and males, with increasing body weight gain speed and fecal gram positive bacteria counts, particularly in females (figures 7D,E). No major difference was observed between genders. 

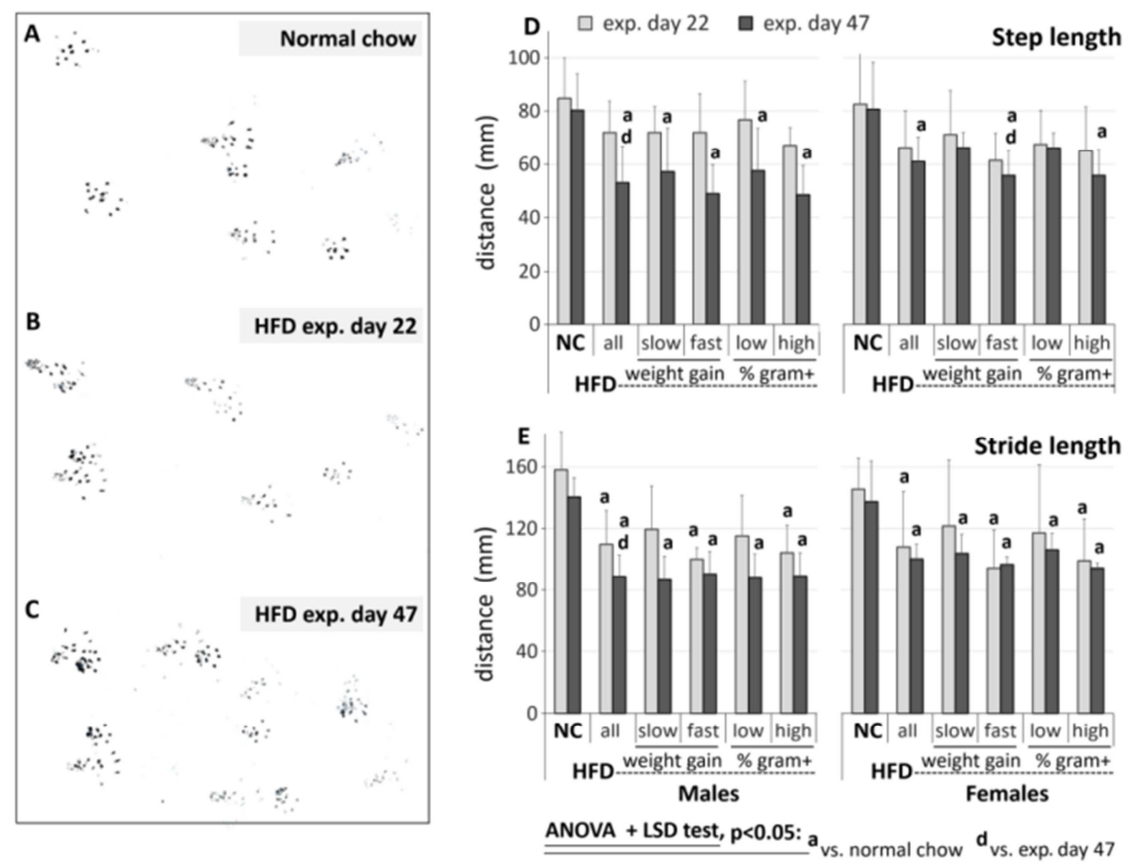

Figure 7. Footprint analysis.

A-C. Footprints of representative cases of normal chow-fed (A), and of a high-fat diet (HFD)-fed animal at exposure day 22, and of the same animal at exposure day 47. Note the shorter steps and the tendency to stand on toes only, with HFD exposure. D,E. Effect of HFD exposure on step (D) and stride length (E). Note the decreases with HFD exposure.

\subsection{Multifactorial Analysis}

The correlations between physiological and behavioral parameters revealed by multifactorial analysis are shown in table 3 .

\subsubsection{Anxiety and Depression Indicators}

Fecal gram positive bacteria counts (increased by HFD) were correlated with 5 out of 7 indicators of anxiety, i.e. headdipping latency (positively), the time spent in the holeboard arena corners or conversely the time spent in the arena center (positively and negatively, respectively), the number of rearing against wall (positively), freezing time (positively), and freezing latency (negatively).

Table 3. Correlations between indicators of physiological and behavioral changes.

\begin{tabular}{|c|c|c|c|c|c|c|c|c|c|}
\hline & & \multicolumn{2}{|c|}{ Fecal gram+ counts } & \multicolumn{2}{|c|}{ Fecal pH } & \multicolumn{2}{|c|}{ Body weight gain } & \multicolumn{2}{|c|}{ Fasting glycemia } \\
\hline & & $r$ & p value & $r$ & p value & $r$ & p value & $r$ & p value \\
\hline \multicolumn{2}{|c|}{ Body weight gain } & $0.61 * * *$ & 0.0002 & $0.81 * * *$ & $2.4 \mathrm{E}-08$ & $\mathrm{n} / \mathrm{a}$ & $\mathrm{n} / \mathrm{a}$ & $0.5 * *$ & 0.004 \\
\hline \multicolumn{2}{|c|}{ Fecal gram + bacteria counts } & $\mathrm{n} / \mathrm{a}$ & $\mathrm{n} / \mathrm{a}$ & $0.53 * *$ & 0.0018 & $0.61 * * *$ & 0.0002 & $0.68 * * *$ & 0.00002 \\
\hline \multicolumn{2}{|l|}{ Fecal pH } & $0.53 * *$ & 0.0018 & $\mathrm{n} / \mathrm{a}$ & $\mathrm{n} / \mathrm{a}$ & $0.81 * * *$ & $2.4 \mathrm{E}-08$ & $0.59 * * *$ & 0.0004 \\
\hline \multicolumn{2}{|c|}{ Fasting glycemia } & $0.68 * * *$ & 0.00002 & $0.59 * * *$ & 0.0004 & $0.5^{* *}$ & 0.004 & $\mathrm{n} / \mathrm{a}$ & $\mathrm{n} / \mathrm{a}$ \\
\hline Pole test & t-turn & $-0.75 * * *$ & 0.00001 & $-0.5 * *$ & 0.0037 & $-0.5 * *$ & 0.004 & $-0.68 * * *$ & 0.00002 \\
\hline \multicolumn{10}{|l|}{ Holeboard test } \\
\hline \multirow{2}{*}{ Distance } & First minute & -0.17 & 0.353 & -0.27 & 0.138 & -0.25 & 0.136 & -0.03 & 0.854 \\
\hline & Last minute & $0.5 * *$ & 0.0039 & 0.34 & 0.057 & $0.39 *$ & 0.048 & $0.38 *$ & 0.034 \\
\hline \multirow{3}{*}{ Head dipping } & latency & $0.52 * *$ & 0.0024 & 0.25 & 0.174 & 0.25 & 0.147 & $0.36^{*}$ & 0.0441 \\
\hline & number & -0.34 & 0.056 & -0.17 & 0.3403 & -0.21 & 0.293 & -0.14 & 0.432 \\
\hline & time & -0.23 & 0.201 & 0.08 & 0.665 & 0.01 & 0.975 & 0.01 & 0.978 \\
\hline \multirow{2}{*}{ Time in } & arena center & $-0.7 * * *$ & 0.0001 & $-0.6 * * *$ & 0.0009 & $-0.6 * * *$ & 0.0003 & $-0.7 * * *$ & 0.00001 \\
\hline & arena angles & $0.6^{* * *}$ & 0.0003 & $0.59 * * *$ & 0.0004 & $0.61 * * *$ & 0.0005 & $0.55^{* *}$ & 0.00102 \\
\hline Rearing & not against wall, $\mathrm{n}$ & -0.28 & 0.116 & -0.29 & 0.1032 & -0.29 & 0.085 & -0.3 & 0.101 \\
\hline \multirow{2}{*}{ Freezing } & latency & $-0.7 * * *$ & 0.00001 & $-0.6 * * *$ & 0.0004 & $-0.6 * * *$ & 0.0007 & $-0.7 * * *$ & 0.0001 \\
\hline & time & $0.39^{*}$ & 0.0267 & $0.51 * *$ & 0.003 & $0.41 *$ & 0.031 & 0.33 & 0.063 \\
\hline \multirow{2}{*}{ Grooming } & latency & $0.44 *$ & 0.0109 & 0.24 & 0.177 & 0.15 & 0.458 & $0.45^{*}$ & 0.0102 \\
\hline & number & $-0.52 * *$ & 0.0023 & -0.32 & 0.07 & -0.27 & 0.101 & $-0.56 * * *$ & 0.0009 \\
\hline
\end{tabular}

n/a: not applicable. Multifactorial analysis: ${ }^{*} \mathrm{p}<0.05,{ }^{* *} \mathrm{p}<0.01,{ }^{* * *} \mathrm{p}<0.001 . \mathrm{N}=32$. 
Fecal $\mathrm{pH}$ was correlated with 3 indicators of anxiety: increase in time spent in the holeboard arena corners, increase in freezing time, and decrease in freezing latency. Correlations of body weight gain and fasting glycemia with indicators of anxiety were comparable. Fasting glycemia was correlated with 4 indicators of anxiety, and less strong than fecal gram positive bacteria counts (table 3), notably: increase in headdipping latency, time spent in the arena corners, number of rearing against wall episodes, and decrease in freezing latency. Body weight gain was correlated with 4 indicators of anxiety: increases in the time spent in the arena corners, number of rearing against wall episodes, freezing time, and decrease in freezing latency, also with less strong correlations than fecal gram positive bacteria counts and fecal $\mathrm{pH}$ (table 3 ). None of these parameters correlated significantly with head dipping number and time (table 3).

In addition, fecal gram positive counts and fasting glycemia, but not body weight gain, were positively correlated with grooming latency and negatively with grooming episode number.

\subsubsection{Motor Function Indicators}

The major motor function indicators determined in the present study were decreases in pole test $\mathrm{t}$-turn and $\mathrm{t}$-time. $\mathrm{T}$ turn and t-time changes were correlated negatively with fecal gram positive counts, fasting glycemia, and in a lesser extent, body weight gain and fecal $\mathrm{pH}$ (table 3 ). Similar, the distance covered in the arena, particularly in the last minute, was also correlated fecal gram positive counts, fasting glycemia, and in a lesser extent, and body weight gain (table 3 ).

\section{Discussion}

The results of the present study suggest that mood alterations, cognitive decline and motor impairment are present in high-fat diet (HFD) induced pre-obese mice, where their severity would correlate with intestinal dysbiosis, and in a lesser extent, body weight gain. The indicators of anxiety observed in pre-obese mice included an increase in the time preferentially spent in the holeboard arena corners compared to the time spent in the central area (safe areas preferred to frightening area [42]), and similar, an increase in the number of episodes of rearing against wall, indicating an increased tendency of pre-obese animal to thigmotaxis, i.e. to move mainly close or in contact to cage walls (safe area) [43, 58]. Anxiety was also suggested by decreased latency and increased length of freezing episodes [59], and increased latency, and decreased time and number of head-dipping episodes [42]. Stress-induced hyperthermia (SIH), a physiological marker of anxiety, was also abnormally increased in pre-obese mice, particularly in those gaining weight faster and with abnormally high fecal gram positive bacteria counts (compared to lean control animals). Overall, the intestinal dysbiosis indicators determined, and particularly fecal gram positive bacteria counts, correlated better with changes indicating anxiety than body weight gain and fasting glycemia, suggesting a more prominent role for intestinal dysbiosis in the pathogenic events triggering and driving anxiety in the pre-obesity context. Lower correlations of anxiety indicators with body weight gain and fasting glycemia may be surprising considering that body weight gain-associates with the positive energy balance that leads to and sustains obesity [12-15], and considering also that deregulation of glycemic control constitutes a major component of metabolic syndrome in obese subjects and animal models $[10,11,60]$. However, we hypothesize that the better correlation of intestinal dysbiosis indicator over alterations of glycemic control and body weight gain may emerge from a driver role for intestinal dysbiosis in obesogenesis and complication development in the obesity context, while the glycemic control and body weight gain may only have an accompanying role, i.e. fueling the obesogenic mechanisms triggered by intestinal dysbiosis. Available evidence supporting this hypothesis include the ability of altered gut microbiota to induce obesity when transplanted in a genetically sensitive healthy lean subject $[18,19,21]$ and gut microbiota role as powerful metabolic and immune modulator [21-23].

Furthermore, indicators of depression, notably increased latency and decreased episode number of grooming, were also observed. Such indicators correlated with fecal gram positive bacteria counts and fasting glycemia, but not with body weight gain, further stressing on a pivotal role for intestinal dysbiosis in anxiety and mood alterations in the pre-obesity context. Although such link was recently hypothesized based on reports of pivotal roles for intestinal dysbiosis in the pathogenesis of anxiety and depression in related metabolic diseases [26, 61], to our knowledge, this is the first report suggesting a link between intestinal dysbiosis and anxiety and mood alterations in the pre-obesity context, at least in a Western diet model.

Interestingly, the indicators of motor dysfunction observed in HFD-induced pre-obese mice (t-turn, t-time in pole test, and footprint analysis) were also correlated with fecal gram positive counts, fasting glycemia, and in a lesser extent, body weight gain. As expected [62, 63], footprint analysis also suggested motor (gait) disturbances in pre-obese animals. Furthermore, cognitive impairment was also suggested by an inversion of exploration and motor activity dominant time from the early to the last minutes spent in the holeboard arena, as suggested by a decrease in the first minute and an increase in the last minute of the distance covered in the arena in HFD-fed animals, compared to normal chow fed. Rodents have a natural tendency to explore novel environments [64, 65], and failure to perform such task readily may result from cognitive deficit [66, 67]. As for mood alterations reported, cognitive and motor dysfunctions observed may emerge, at least partly, from immune-to-brain signaling triggered by intestinal dysbiosis-induced local and systemic inflammation $[26,68,69]$.

To verify this hypothesis, we have assessed inflammation indicators and bacterial infiltration in the jejunum. Infiltrating 
bacteria, bacilli mainly, were found in the jejunal mucosa as well as signs of non-specific inflammation (fluid exudate and epithelial cell degeneration...), and an increased expression of CXCL10, a marker of sustained inflammation [70, 71]. In addition, liver and gastro-intestinal tract weight were increased, indicating accumulations of fluids and fat in these tissues [72-74]. These alterations were particularly marked in HFD-fed animals gaining weight faster, and more in females than males. Considering that females that gained weight significantly faster than males also had more marked alterations in dysbiosis indicators, including fecal $\mathrm{pH}$ and fecal gram positive bacteria counts, and, given that as males, females with low fecal bacteria counts displayed only a slow weight gain, it appears that fast weight gain may result from intestinal dysbiosis induced by subchronic HFD exposure [20, 21]. Besides gender physiological specificities, differences in host genetic susceptibility to dysbiosis $[18,19]$, suggested by high inter-individual differences in the response to HFD subchronic exposure in males, probably contribute also to lower weight gain observed in males in the present study.

The present study mainly addressed clinical indicators of intestinal dysbiosis with the final objective of comparing their occurrence in animals with marked behavioral changes. Studies with more advanced methods such as targeted qPCR and 16s rRNA sequencing should be carried out in HFD preobese mice for insights in more complex aspects of intestinal dysbiosis. Notably, unraveling the specific bacterial species whose changes associate with behavioral alterations could be of particular interest for metabolic syndrome treatment. The present findings should be assessed in obese individuals and patients with metabolic syndrome-associated diseases, considering the potential for early detection of monitoring of various obesity-related comorbidities.

\section{Conclusion}

We have used a high-fat diet (HFD) to induce pre-obesity in mice, a model of rich diet-induced obesity. The behavioral tests performed in these animals suggested anxiety, depression, and cognitive and motor impairment. Such central nervous system functional alterations were more marked in females, particularly in animals with marked alterations in intestinal dysbiosis indicators, and in a lesser extent, also in animals with increased fasting glycemia or those displaying a relatively fast weight gain. Overall, our findings point out intestinal dysbiosis as a major player in the pathogenesis of obesity-related comorbidities involving the brain, as hallmarks of strong intestinal dysbiosis appeared concomitantly and correlated, better than any other parameter, with indicators of nervous system functional alterations observed in pre-obese mice, whose metabolic syndrome is less marked than obese animals. Future study addressing the interaction of altered gut microbiota with the other players active in the obesity context, in particular metabolic syndrome components, may provide new insights in the mechanisms driving and sustaining obesogenesis and the development of obesity-related comorbidities.

\section{Acknowledgments}

The present study was supported by the Research Deanship and the College of Applied Medical Sciences, Qassim University, Buraydah, Saudi Arabia. Authors thank the colleagues of their respective institutions for their support and for manuscript proofreading.

\section{References}

[1] Lake, A. A.; Townshend, T.; Alvanides, S.; Stamp, E.; Adamson, A. J. Diet, physical activity, sedentary behaviour and perceptions of the environment in young adults. J. Hum. Nutr. Diet. 2009, 22:444-454.

[2] Wang, Y.; Jahns, L.; Tussing-Humphreys, L.; Xie, B.; Rockett, H.; Liang, H.; Johnson, L. Dietary intake patterns of lowincome urban african-american adolescents. J. Am. Diet. Assoc. 2010, 110:1340-1345.

[3] McCarthy, S. Weekly patterns, diet quality and energy balance. Physiol Behav. 2014, 134:55-59.

[4] McElroy, S. L.; Kotwal, R.; Malhotra, S.; Nelson, E. B.; Keck, P. E.; Nemeroff, C. B. Are mood disorders and obesity related? A review for the mental health professional. J. Clin. Psychiatry 2004, 65:634-51, quiz.

[5] Bodenlos, J. S.; Lemon, S. C.; Schneider, K. L.; August, M. A.; Pagoto, S. L. Associations of mood and anxiety disorders with obesity: comparisons by ethnicity. J. Psychosom. Res. 2011, 71:319-324.

[6] Nguyen, J. C.; Killcross, A. S.; Jenkins, T. A. Obesity and cognitive decline: role of inflammation and vascular changes. Front Neurosci. 2014, 8:375.

[7] Miller, A. A.; Spencer, S. J. Obesity and neuroinflammation: a pathway to cognitive impairment. Brain Behav. Immun. 2014, 42:10-21.

[8] Gentier, I.; D'Hondt, E.; Shultz, S.; Deforche, B.; Augustijn, M.; Hoorne, S.; Verlaecke, K.; De, B., I; Lenoir, M. Fine and gross motor skills differ between healthy-weight and obese children. Res. Dev. Disabil. 2013, 34:4043-4051.

[9] D'Hondt, E.; Deforche, B.; Gentier, I.; Verstuyf, J.; Vaeyens, R.; De, B., I; Philippaerts, R.; Lenoir, M. A longitudinal study of gross motor coordination and weight status in children. Obesity. (Silver. Spring) 2014, 22:1505-1511.

[10] Byard, R. W. Forensic issues at the extremes of weight: From frailty syndrome through frail obesity to morbid obesity. J. Forensic Leg. Med. 2015, 35:38-39.

[11] Mauvais-Jarvis, F. Sex differences in metabolic homeostasis, diabetes, and obesity. Biol. Sex Differ. 2015, 6:14.

[12] Ahima, R. S.; Qi, Y.; Singhal, N. S. Adipokines that link obesity and diabetes to the hypothalamus. Prog. Brain Res. 2006, 153:155-174.

[13] Badman, M. K.; Flier, J. S. The adipocyte as an active participant in energy balance and metabolism. Gastroenterology 2007, 132:2103-2115. 
[14] Rummel, C.; Inoue, W.; Poole, S.; Luheshi, G. N. Leptin regulates leukocyte recruitment into the brain following systemic LPS-induced inflammation. Mol. Psychiatry 2010, $15: 523-534$

[15] Farooqui, A. A.; Farooqui, T.; Panza, F.; Frisardi, V. Metabolic syndrome as a risk factor for neurological disorders. Cell Mol. Life Sci. 2012, 69:741-762.

[16] Shawcross, D. L. Is it time to target gut dysbiosis and immune dysfunction in the therapy of hepatic encephalopathy? Expert. Rev. Gastroenterol. Hepatol. 2015, 9:539-542.

[17] Parekh, P. J.; Balart, L. A.; Johnson, D. A. The Influence of the Gut Microbiome on Obesity, Metabolic Syndrome and Gastrointestinal Disease. Clin. Transl. Gastroenterol. 2015, 6:e91.

[18] Anhe, F. F.; Varin, T. V.; Le, B. M.; Desjardins, Y.; Levy, E.; Roy, D.; Marette, A. Gut Microbiota Dysbiosis in ObesityLinked Metabolic Diseases and Prebiotic Potential of Polyphenol-Rich Extracts. Curr. Obes. Rep. 2015.

[19] Ussar, S.; Griffin, N. W.; Bezy, O.; Fujisaka, S.; Vienberg, S.; Softic, S.; Deng, L.; Bry, L.; Gordon, J. I.; Kahn, C. R. Interactions between Gut Microbiota, Host Genetics and Diet Modulate the Predisposition to Obesity and Metabolic Syndrome. Cell Metab 2015, 22:516-530.

[20] Marteau, P.; Chaput, U. Bacteria as trigger for chronic gastrointestinal disorders. Dig. Dis. 2011, 29:166-171.

[21] Underwood, M. A. Intestinal dysbiosis: novel mechanisms by which gut microbes trigger and prevent disease. Prev. Med. 2014, 65:133-137.

[22] Bleich, A.; Fox, J. G. The Mammalian Microbiome and Its Importance in Laboratory Animal Research. ILAR. J. 2015, 56:153-158.

[23] Marchesi, J. R.; Adams, D. H.; Fava, F.; Hermes, G. D.; Hirschfield, G. M.; Hold, G.; Quraishi, M. N.; Kinross, J.; Smidt, H.; Tuohy, K. M.; Thomas, L. V.; Zoetendal, E. G.; Hart, A. The gut microbiota and host health: a new clinical frontier. Gut 2015.

[24] Mehal, W. Z. The Gordian Knot of dysbiosis, obesity and NAFLD. Nat. Rev. Gastroenterol. Hepatol. 2013, 10:637-644.

[25] Serino, M.; Blasco-Baque, V.; Nicolas, S.; Burcelin, R. Far from the eyes, close to the heart: dysbiosis of gut microbiota and cardiovascular consequences. Curr. Cardiol. Rep. 2014, 16:540.

[26] Castanon, N.; Luheshi, G.; Laye, S. Role of neuroinflammation in the emotional and cognitive alterations displayed by animal models of obesity. Front Neurosci. 2015, 9:229.

[27] Gorky, J.; Schwaber, J. The role of the gut-brain axis in alcohol use disorders. Prog. Neuropsychopharmacol. Biol. Psychiatry 2015.

[28] Logan, A. C. Dysbiotic drift: mental health, environmental grey space, and microbiota. J. Physiol Anthropol. 2015, 34:23.

[29] Bienenstock, J.; Kunze, W.; Forsythe, P. Microbiota and the gut-brain axis. Nutr. Rev. 2015, 73 Suppl 1:28-31.

[30] van Esterik, J. C.; Dolle, M. E.; Lamoree, M. H.; van Leeuwen, S. P.; Hamers, T.; Legler, J.; van der Ven, L. T. Programming of metabolic effects in $\mathrm{C} 57 \mathrm{BL} / 6 \mathrm{JxFVB}$ mice by exposure to bisphenol A during gestation and lactation. Toxicology 2014, 321:40-52.

[31] Kim, E.; Kim, E. J.; Seo, S. W.; Hur, C. G.; McGregor, R. A.; Choi, M. S. Meta-review of protein network regulating obesity between validated obesity candidate genes in the white adipose tissue of high-fat diet-induced obese C57BL/6J mice. Crit Rev. Food Sci. Nutr. 2014, 54:910-923.

[32] Katsiki, N.; Mikhailidis, D. P. Emerging Vascular Risk Factors in Women: any differences from men? Curr. Med. Chem. 2015.

[33] Nakamura, A.; Terauchi, Y. Lessons from mouse models of high-fat diet-induced NAFLD. Int. J. Mol. Sci. 2013, 14:21240-21257.

[34] Morselli, E.; Frank, A. P.; Palmer, B. F.; Rodriguez-Navas, C.; Criollo, A.; Clegg, D. J. A sexually dimorphic hypothalamic response to chronic high-fat diet consumption. Int. J. Obes. (Lond) 2015.

[35] Carter, R.; Mouralidarane, A.; Soeda, J.; Ray, S.; Pombo, J.; Saraswati, R.; Novelli, M.; Fusai, G.; Rappa, F.; Saracino, C.; Pazienza, V.; Poston, L.; Taylor, P. D.; Vinciguerra, M.; Oben, J. A. Non-alcoholic fatty pancreas disease pathogenesis: a role for developmental programming and altered circadian rhythms. PLoS. One. 2014, 9:e89505.

[36] Blackmore, H. L.; Niu, Y.; Fernandez-Twinn, D. S.; TarryAdkins, J. L.; Giussani, D. A.; Ozanne, S. E. Maternal dietinduced obesity programs cardiovascular dysfunction in adult male mouse offspring independent of current body weight. Endocrinology 2014, 155:3970-3980.

[37] De Winther, M. P.; Gijbels, M. J.; Van Dijk, K. W.; Havekes, L. M.; Hofker, M. H. Transgenic mouse models to study the role of the macrophage scavenger receptor class A in atherosclerosis. Int. J. Tissue React. 2000, 22:85-91.

[38] Doggrell, S. Do peroxisome proliferation receptor-gamma antagonists have clinical potential as combined antiobesity and antidiabetic drugs? Expert. Opin. Investig. Drugs 2003, 12:713-716.

[39] Hirotani, Y.; Ozaki, N.; Tsuji, Y.; Urashima, Y.; Myotoku, M. Effects of eicosapentaenoic acid on hepatic dyslipidemia and oxidative stress in high fat diet-induced steatosis. Int. J. Food Sci. Nutr. 2015, 66:569-573.

[40] Shi, L. L.; Li, Y.; Wang, Y.; Feng, Y. MDG-1, an Ophiopogon polysaccharide, regulate gut microbiota in high-fat dietinduced obese C57BL/6 mice. Int. J. Biol. Macromol. 2015, 81:576-583

[41] Portaluppi, F.; Smolensky, M. H.; Touitou, Y. Ethics and methods for biological rhythm research on animals and human beings. Chronobiol. Int. 2010, 27:1911-1929.

[42] Brown, G. R.; Nemes, C. The exploratory behaviour of rats in the hole-board apparatus: is head-dipping a valid measure of neophilia? Behav. Processes 2008, 78:442-448.

[43] Lamprea, M. R.; Cardenas, F. P.; Setem, J.; Morato, S. Thigmotactic responses in an open-field. Braz. J. Med. Biol. Res. 2008, 41:135-140.

[44] Van der Heyden, J. A.; Zethof, T. J.; Olivier, B. Stress-induced hyperthermia in singly housed mice. Physiol Behav. 1997, 62:463-470 
[45] Olivier, B.; Zethof, T.; Pattij, T.; van, B. M.; van, O. R.; Leahy, C.; Oosting, R.; Bouwknecht, A.; Veening, J.; van der Gugten, J.; Groenink, L. Stress-induced hyperthermia and anxiety: pharmacological validation. Eur. J. Pharmacol. 2003, 463:117-132.

[46] Adriaan, B. J.; Olivier, B.; Paylor, R. E. The stress-induced hyperthermia paradigm as a physiological animal model for anxiety: a review of pharmacological and genetic studies in the mouse. Neurosci. Biobehav. Rev. 2007, 31:41-59.

[47] Vinkers, C. H.; van Bogaert, M. J.; Klanker, M.; Korte, S. M.; Oosting, R.; Hanania, T.; Hopkins, S. C.; Olivier, B.; Groenink, L. Translational aspects of pharmacological research into anxiety disorders: the stress-induced hyperthermia (SIH) paradigm. Eur. J. Pharmacol. 2008, 585:407-425.

[48] Groenink, L.; Vinkers, C.; van, O. R.; Olivier, B. Models of anxiety: stress-induced hyperthermia (SIH) in singly housed mice. Curr. Protoc. Pharmacol. 2009, Chapter 5:Unit.

[49] Wieronska, J. M.; Stachowicz, K.; Branski, P.; PaluchaPoniewiera, A.; Pilc, A. On the mechanism of antihyperthermic effects of LY379268 and LY487379, group II mGlu receptors activators, in the stress-induced hyperthermia in singly housed mice. Neuropharmacology 2012, 62:322-331.

[50] Kistemaker, J. A.; Den Hartog, E. A.; Daanen, H. A. Reliability of an infrared forehead skin thermometer for core temperature measurements. J. Med. Eng Technol. 2006, $30: 252-261$

[51] Hishimura, Y.; Itoh, K. Effect of social interaction on skin temperature in mice. Shinrigaku Kenkyu 2009, 80:152-158.

[52] Bach, A. J.; Stewart, I. B.; Minett, G. M.; Costello, J. T. Does the technique employed for skin temperature assessment alter outcomes? A systematic review. Physiol Meas. 2015, 36:R27R51.

[53] Ansley, L.; Marvin, G.; Sharma, A.; Kendall, M. J.; Jones, D. A.; Bridge, M. W. The effects of head cooling on endurance and neuroendocrine responses to exercise in warm conditions. Physiol Res. 2008, 57:863-872.

[54] Schlader, Z. J.; Perry, B. G.; Jusoh, M. R.; Hodges, L. D.; Stannard, S. R.; Mundel, T. Human temperature regulation when given the opportunity to behave. Eur. J. Appl. Physiol 2013, 113:1291-1301.

[55] Paylor, R.; Nguyen, M.; Crawley, J. N.; Patrick, J.; Beaudet, A.; Orr-Urtreger, A. Alpha7 nicotinic receptor subunits are not necessary for hippocampal-dependent learning or sensorimotor gating: a behavioral characterization of Acra7deficient mice. Learn. Mem. 1998, 5:302-316.

[56] Bouet, V.; Freret, T.; Toutain, J.; Divoux, D.; Boulouard, M.; Schumann-Bard, P. Sensorimotor and cognitive deficits after transient middle cerebral artery occlusion in the mouse. Exp. Neurol. 2007, 203:555-567.

[57] Carter, R. J.; Lione, L. A.; Humby, T.; Mangiarini, L.; Mahal, A.; Bates, G. P.; Dunnett, S. B.; Morton, A. J. Characterization of progressive motor deficits in mice transgenic for the human Huntington's disease mutation. J. Neurosci. 1999, 19:32483257.

[58] Simon, P.; Dupuis, R.; Costentin, J. Thigmotaxis as an index of anxiety in mice. Influence of dopaminergic transmissions. Behav. Brain Res. 1994, 61:59-64.
[59] Depino, A. M.; Gross, C. Simultaneous assessment of autonomic function and anxiety-related behavior in BALB/c and C57BL/6 mice. Behav. Brain Res. 2007, 177:254-260.

[60] Yan, W. J.; Mu, Y.; Yu, N.; Yi, T. L.; Zhang, Y.; Pang, X. L.; Cheng, D.; Yang, J. Protective effects of metformin on reproductive function in obese male rats induced by high-fat diet. J. Assist. Reprod. Genet. 2015, 32:1097-1104.

[61] Scalera, A.; Di Minno, M. N.; Tarantino, G. What does irritable bowel syndrome share with non-alcoholic fatty liver disease? World J. Gastroenterol. 2013, 19:5402-5420.

[62] Riddiford-Harland, D. L.; Steele, J. R.; Storlien, L. H. Does obesity influence foot structure in prepubescent children? Int. J. Obes. Relat Metab Disord. 2000, 24:541-544.

[63] Sarkar, A.; Singh, M.; Bansal, N.; Kapoor, S. Effects of obesity on balance and gait alterations in young adults. Indian J. Physiol Pharmacol. 2011, 55:227-233.

[64] Valles, A.; Boender, A. J.; Gijsbers, S.; Haast, R. A.; Martens, G. J.; de, W. P. Genomewide analysis of rat barrel cortex reveals time- and layer-specific mRNA expression changes related to experience-dependent plasticity. J. Neurosci. 2011, 31:6140-6158.

[65] d'Isa, R.; Brambilla, R.; Fasano, S. Behavioral methods for the study of the Ras-ERK pathway in memory formation and consolidation: passive avoidance and novel object recognition tests. Methods Mol. Biol. 2014, 1120:131-156.

[66] Gobbo, O. L.; O'Mara, S. M. Impact of enriched-environment housing on brain-derived neurotrophic factor and on cognitive performance after a transient global ischemia. Behav. Brain Res. 2004, 152:231-241.

[67] Vaseghi, G.; Rabbani, M.; Hajhashemi, V. The CB(1) receptor antagonist, AM281, improves recognition loss induced by naloxone in morphine withdrawal mice. Basic Clin. Pharmacol. Toxicol. 2012, 111:161-165.

[68] Amar, S.; Zhou, Q.; Shaik-Dasthagirisaheb, Y.; Leeman, S. Diet-induced obesity in mice causes changes in immune responses and bone loss manifested by bacterial challenge. Proc. Natl. Acad. Sci. U. S. A 2007, 104:20466-20471.

[69] Andre, C.; Dinel, A. L.; Ferreira, G.; Laye, S.; Castanon, N. Diet-induced obesity progressively alters cognition, anxietylike behavior and lipopolysaccharide-induced depressive-like behavior: focus on brain indoleamine 2,3-dioxygenase activation. Brain Behav. Immun. 2014, 41:10-21.

[70] Crescioli, C. Chemokines and transplant outcome. Clin. Biochem. 2015.

[71] Van Raemdonck, K.; Van den Steen, P. E.; Liekens, S.; Van Damme, J.; Struyf, S. CXCR3 ligands in disease and therapy. Cytokine Growth Factor Rev. 2015, 26:311-327.

[72] Helajarvi, H.; Pahkala, K.; Heinonen, O. J.; Juonala, M.; Oikonen, M.; Tammelin, T.; Hutri-Kahonen, N.; Kahonen, M.; Lehtimaki, T.; Mikkila, V.; Viikari, J.; Raitakari, O. T. Television viewing and fatty liver in early midlife. The Cardiovascular Risk in Young Finns Study. Ann. Med. 2015:18.

[73] Fabbrini, E.; Tiemann, L. C.; Love-Gregory, L.; Okunade, A. L.; Yoshino, M.; Fraterrigo, G.; Patterson, B. W.; Klein, S. Physiological Mechanisms of Weight-gain Induced Steatosis in People with Obesity. Gastroenterology 2015. 
[74] Bottino, C.; Vazquez, M.; Devesa, V.; Laforenza, U. Impaired aquaporins expression in the gastrointestinal tract of rat after mercury exposure. J. Appl. Toxicol. 2015. 